\title{
Massless black holes and black rings as effective geometries of the D1-D5 system
}

\author{
Vijay Balasubramanian ${ }^{1}$, Per Kraus ${ }^{2}$ and Masaki Shigemori ${ }^{3}$ \\ ${ }^{1}$ David Rittenhouse Laboratories, University of Pennsylvania, Philadelphia, PA 19104, USA \\ 2 Department of Physics and Astronomy, UCLA, Los Angeles, CA 90095-1547, USA \\ ${ }^{3}$ California Institute of Technology 452-48, Pasadena, CA 91125, USA \\ E-mail: vijay@physics.upenn.edu,pkraus@physics.ucla.edu and shige@theory.caltech.edu
}

Received 13 September 2005

Published 27 October 2005

Online at stacks.iop.org/CQG/22/4803

\begin{abstract}
We compute correlation functions in the $\mathrm{AdS}_{3} / \mathrm{CFT}_{2}$ correspondence to study the emergence of effective spacetime geometries describing complex underlying microstates. The basic argument is that almost all microstates of fixed charges lie close to certain 'typical' configurations. These give a universal response to generic probes, which is captured by an emergent geometry. The details of the microstates can only be observed by atypical probes. We compute two-point functions in typical ground states of the Ramond sector of the D1-D5 CFT, and compare with bulk two-point functions computed in asymptotically $\mathrm{AdS}_{3}$ geometries. For large central charge (which leads to a good semiclassical limit), and sufficiently small time separation, a typical Ramond ground state of vanishing $R$-charge has the $M=0 \mathrm{BTZ}$ black hole as its effective description. At large time separation this effective description breaks down. The CFT correlators we compute take over, and give a response whose details depend on the microstate. We also discuss typical states with nonzero $R$-charge, and argue that the effective geometry should be a singular black ring. Our results support the argument that a black hole geometry should be understood as an effective coarse-grained description that accurately describes the results of certain typical measurements, but breaks down in general.
\end{abstract}

PACS numbers: $04.70 .-\mathrm{s}, 11.25 . \mathrm{Tq}, 11.25 . \mathrm{Uv}$

\section{Introduction}

The AdS/CFT correspondence [1-3] has provided a detailed connection between black holes and conformal field theories. Although it is sometimes said that this solves the conceptual puzzles associated with black hole physics, in fact we still do not understand the connection well enough to see explicitly how all these puzzles are resolved. 
In using the AdS/CFT correspondence in the context of black holes one typically compares a thermal ensemble in the CFT to a semiclassical black hole geometry in the bulk. In this way, it is possible to compute and compare quantities such as the entropy of the system and correlation functions of fields/operators [4-12]. Recent work [13-21] has shown that in some cases one can do even better by extending this relation to the regime where the bulk geometry receives large corrections from higher derivative string and loop effects.

In the CFT, it is manifest that the thermal ensemble corresponds to a weighted collection of individual microstates. Instead of considering such an ensemble, there is nothing to prevent one from choosing a particular microstate and computing correlation functions in that state. On general grounds, if one is working at large $N$ then correlation functions of 'typical' operators computed in a 'typical' state will be approximated to excellent accuracy by the same correlators computed in the thermal ensemble. This is just the same as saying that realistic isolated systems, i.e., a large number of molecules in a sealed box, are in some particular quantum mechanical microstate at a given time, yet can be accurately studied by the methods of statistical mechanics and thermodynamics.

The most natural interpretation of the AdS/CFT correspondence is that there is a oneto-one correspondence between bulk and boundary states. In particular, one expects this statement to hold even in the range of parameters where black holes are allowed. Precisely what the bulk microstates should look like is unclear at this point in time. Mathur [22] has conjectured that these microstates correspond to bulk geometries (in general these might be classically singular or have large quantum fluctuations) without horizons, and the evidence for this conjecture includes [22-29]. Just as in the CFT, one is led to believe that if one chooses a typical such bulk state then with respect to typical measurements it will look like the usual black hole geometry.

An example of an atypical measurement is one which extends over a very long time interval. As originally emphasized by Maldacena [4], at late times correlators computed in the semiclassical black hole geometry decay to zero, while in the CFT they exhibit a quasi-periodic behaviour. The key difference is that the semiclassical black hole geometry has a continuous spectrum due to the presence of the horizon, while the CFT has a discrete spectrum. This distinction is insignificant for short times or at high energy, but becomes important in the opposite regime. Further work [9, 11] in the context of BTZ black holes [30] strongly indicates that also summing over the $S L(2, \mathbb{Z})$ images of the black hole (which includes global $\mathrm{AdS}_{3}$ ) can prevent the correlators from decaying to zero, but cannot account for the quasi-periodicity. Presumably, this suggests that we should instead be considering the actual microstate geometries dual to the individual CFT microstates if we want to correctly account for detailed properties such as the late time behaviour of correlators. The analogy with molecules in a box is again helpful: while a coarse-grained effective description accurately describes most properties of the system, in order to recover the quasi-periodicity of late time correlators one needs to return to the fundamental molecular description.

Recently, some of these issues have been discussed in the context of the $\mathrm{AdS}_{5} / \mathrm{CFT}_{4}$ correspondence for half-BPS states [31-33]. It was argued in [34] that typical large-charge half-BPS microstates that are incipient black holes have a spacetime description as a quantum 'foam', the precise details of which are almost invisible to almost all probes. This gave rise to effective singular descriptions of underlying smooth quantum states [34]. (Other perspectives on these issues have appeared in [35].) In the present paper, we will study the D1-D5 system on $T^{4}$, since this provides the simplest link between black holes and CFT. We will also set to zero the momentum $P$, so that we just work with the Ramond-Ramond ground states of the system. This example is of interest for several reasons. The system has a large ground state degeneracy corresponding to an entropy $S=2 \pi \sqrt{2} \sqrt{N_{1} N_{5}}$, and so should have some of the 
properties of a black hole ${ }^{4}$. A large class of microstate geometries for this system is known. They correspond to configurations in which the D1 and D5 branes expand into a Kaluza-Klein monopole supertube [36]. The shape of the supertube encodes the details of the microstate. For example, the maximally $R$-charged microstate corresponds to a circular supertube. On the other hand, a typical state corresponds to the supertube taking a complicated random walk shape localized near the origin. This leads to a strongly curved supergravity solution whose existence is inferred via extrapolation from the weakly curved geometries described by smooth curves of large size.

We will compute correlation functions of certain operators in typical states of the D1-D5 system. As we have already discussed, at large $N=N_{1} N_{5}$ the expectation is that these correlators should coincide with bulk correlators computed in some effective geometry. This effective geometry is analogous to the black hole geometry in the case of the D1-D5-P system. As we will see, the effective geometry that emerges depends on the $R$-charge of the underlying state. If the $R$-charge vanishes, the emergent geometry is the massless BTZ [30] black hole; or equivalently, $\mathrm{AdS}_{3}$ in Poincaré coordinates with a spatial direction periodically identified. This is often referred to as the 'naive' geometry representing the RR ground states. It can be obtained by contracting the KK-monopole supertube to zero size. No individual microstate corresponds to this geometry; rather, in the large $N$ limit, this geometry encodes the universal response of generic finite-time correlation functions in the underlying microstate. This effective geometry exhibits a continuous spectrum just like the black hole, and so bulk correlators decay to zero at late times. But in this case we can also show that the exact late time correlation functions show quasi-periodic behaviour demonstrating that the effective description breaks down at large times, and should be replaced by the exact microstate geometries.

We would like to emphasize that our approach based on computing correlation functions allows us to derive the effective geometries corresponding to CFT states, rather than assuming the (highly plausible!) map between states and KK-monopole supertube profiles. A correlation function based approach is also necessary if one wishes to make statements about the geometry at the string or Planck scale, since the existing map between states and geometries is only valid at the level of two-derivative supergravity.

We also consider typical states of nonzero $R$-charge, and find some new features. For sufficiently large charge, the CFT undergoes a form of Bose-Einstein condensation. The state effectively splits into two components, one carrying the $R$-charge but no entropy, and the other carrying no $R$-charge and all the entropy. The correlation functions we compute then become a sum of two terms with contributions from each of the two components. The result looks effectively like a superposition of correlators computed in the massless BTZ space and in the maximal $R$-charge Ramond vacuum, namely globals $\mathrm{AdS}_{3}$ with a Wilson line [36-38]. On the other hand, we are able to derive a prediction for the effective bulk geometry with nonzero $R$-charge by explicitly constructing the typical microstate geometry and coarse-graining it. In effect, this amounts to adding fluctuations on top of the circular supertube solution and then averaging over these fluctuations in the large $N$ limit. The predicted effective geometry for nonzero $R$-charge turns out to be a singular black ring solution. It would interesting to establish the connection between this prediction and the 'superposition' of geometries derived from the CFT correlators.

The ultimate goal of this sort of investigation is to see a macroscopic semiclassical black hole geometry emerging from correlators computed in a typical state of a large $N$ CFT. This requires knowing how the presence of a black hole manifests itself in terms of correlators.

\footnotetext{
4 In the case of the D1-D5 system on K3 it has been shown that higher curvature terms indeed lead to an event horizon whose entropy coincides with that in the CFT $[15,16]$. Whether this also happens in the $T^{4}$ case is unclear [13-21].
} 
Let us note two criteria. First, the correlators should correspond to a well-defined classical geometry, rather than a strongly fluctuating superposition. Second, correlators should fall to zero at late times (in the large $N$ limit), reflecting the presence of a horizon. Here we observe that the effective geometry emerging from our computations satisfies these two properties, and so we can claim to be seeing some black hole-like properties. For a large black hole one would like to do better and reproduce the most important property of all-that of complete absorption of high energy particles impinging on the horizon, but this goes beyond what we can do here.

The remainder of this paper is organized as follows. In section 2 we review the physics of the D1-D5 system, summarizing the relevant details of the D1-D5 CFT, the map between Ramond ground states and microstate geometries, and the computation of two-point correlation functions for massless scalars in the black hole spacetimes. A more extensive review explaining details appears in appendix A. In section 3, we construct the typical Ramond ground states of the D1-D5 CFT that have fixed $R$-charges. In section 4 we derive the effective geometries describing finite-time correlation functions computed in these microstates. The basic technique is to compute and analyse the two-point correlator in the typical states constructed in section 3 . In section 5 we conclude. Appendix B gives additional details about typical states with nonzero $R$-charge.

\section{The D1-D5 system and its geometric dual}

\subsection{The D1-D5 CFT and its Ramond sector ground states}

Consider type IIB string theory on $S^{1} \times T^{4}$ with $N_{1}$ D1-branes and $N_{5}$ D5-branes. The D1-branes are wound on $S^{1}$ and the D5-branes are wrapped on $S^{1} \times T^{4}$. At low energies, the worldvolume dynamics of the branes is given by an $\mathcal{N}=(4,4)$ supersymmetric sigma model whose target space is the symmetric product $\mathcal{M}_{0}=\left(T^{4}\right)^{N} / S_{N}$, where $S_{N}$ is the permutation group of $\operatorname{order} N$ [39-42]. Here we set

$$
N \equiv N_{1} N_{5}
$$

More precisely, $\mathcal{M}_{0}$ is the so-called orbifold point in a family of CFTs which are regained by turning on certain marginal deformations of the sigma model on $\mathcal{M}_{0}$. At the orbifold point the CFT becomes free. The D1-D5 CFT is dual to type IIB string theory on $\mathrm{AdS}_{3} \times S^{3} \times T^{4}$, which is the near-horizon limit of the D1-D5 brane system. The $\mathrm{AdS}_{3}$ length scale is given by $\ell \sim N^{1 / 4}$. To have a large, weakly coupled, $\mathrm{AdS}_{3}$ space, $N$ must be large and the CFT must be deformed far from the orbifold point. This situation is familiar in the $\mathrm{AdS}_{5} / \mathrm{SYM}_{4}$ duality, where the SYM theory becomes free at a special point $\left(g_{\mathrm{YM}}=0\right)$ in the moduli space, but in order for it to correspond to a semiclassical gravity one has to turn on the coupling $g_{\mathrm{YM}}$. The orbifold point is the analogue of the free SYM. In the following, we will consider the orbifold point of the D1-D5 CFT, so one should bear in mind that exact agreement with computations in supergravity is not expected in all cases, although some protected BPS quantities can be computed exactly.

According to the AdS/CFT correspondence, every pure state of the D1-D5 CFT is dual to a pure state of string theory in $\mathrm{AdS}_{3} \times S^{3} \times T^{4}$. Here, we are interested in understanding how black holes emerge as the effective spacetime description of underlying pure states in gravity. The only black holes in $\mathrm{AdS}_{3}$ gravity with standard boundary conditions are the BTZ solutions [30]. The supersymmetric versions of these spacetimes have periodic boundary conditions for fermions around the asymptotic circle in the $\mathrm{AdS}_{3}$ geometry and thus appear in the Ramond sector of the theory. Furthermore, the lightest of the black holes, the BPS 
massless solution, has the quantum numbers of a ground state in the Ramond sector of the dual CFT [43]. For these reasons, we will concentrate on the Ramond ground states of the D1-D5 CFT henceforth. Powerful techniques to study these ground states are available at the orbifold point of the CFT.

The D1-D5 CFT and the construction of the Ramond ground states is reviewed in detail in appendix A. For the moment, the following facts are sufficient. At the orbifold point we are dealing with an $\mathcal{N}=(4,4)$ SCFT on the target space $\mathcal{M}_{0}=\left(T^{4}\right)^{N} / S_{N}$. This theory has an $S U(2)_{R} \times \widetilde{S U(2)_{R}} R$-symmetry, which originates from the $S O$ (4) rotational symmetry transverse to the D1-D5 worldvolume. There is another global $S U(2)_{I} \times \widehat{S U(2)_{I}}$ which is broken by the toroidal identifications in $T^{4}$, but can be used for classifying states anyway. We will label the charges under these symmetries as

$$
\left(J_{R}^{3}, \tilde{J}_{R}^{3}\right)=\left(\frac{s}{2}, \frac{\tilde{s}}{2}\right) \quad \text { and } \quad\left(I^{3}, \tilde{I}^{3}\right)=\left(\frac{\alpha}{2}, \frac{\tilde{\alpha}}{2}\right)
$$

with $s, \tilde{s}, \alpha, \tilde{\alpha}= \pm 1$. The CFT has a collection of twist fields $\sigma_{n}$, which cyclically permute $n \leqslant N$ copies of the CFT on a single $T^{4}$. One can think of these operators as creating winding sectors of the worldsheet that wind over the different copies of the torus. The product of twist operators is also a twist operator. The elementary bosonic operators of twist $n$ carry either $S U(2)_{R} \times{\widehat{S U(2)_{R}}}_{R}$ or $\widehat{S U(2)_{I}}$ charges $\left(\sigma_{n}^{s \tilde{s}}\right.$ or $\left.\sigma_{n}^{\widetilde{\alpha} \widetilde{\beta}}\right)$, while the elementary fermionic twist operators are charged under $S U(2)_{R} \times{\widehat{S U(2)_{I}}}_{I}$ or $\widehat{S U(2)_{I}} \times \widehat{S U(2)_{R}}\left(\tau_{n}^{s \widetilde{\alpha}}\right.$ or $\left.\tau_{n}^{\widetilde{\alpha s}}\right)$. A general Ramond sector ground state is constructed by multiplying together elementary bosonic and fermionic twist operators to achieve a total twist of $N=N_{1} N_{5}$ :

$$
\begin{aligned}
& \sigma=\prod_{n, \mu}\left(\sigma_{n}^{\mu}\right)^{N_{n \mu}}\left(\tau_{n}^{\mu}\right)^{N_{n \mu}^{\prime}}, \\
& \sum_{n, \mu} n\left(N_{n \mu}+N_{n \mu}^{\prime}\right)=N, \quad N_{n \mu}=0,1,2, \ldots, \quad N_{n \mu}^{\prime}=0,1 .
\end{aligned}
$$

Here $\sigma_{n}^{\mu}$ and $\tau_{n}^{\mu}$ are the constituent elementary twist operators, and $\mu=1, \ldots, 8$ labels their possible polarizations $(\mu=(s, \widetilde{s}),(\widetilde{\alpha}, \widetilde{\beta})$ for bosons, and $\mu=(s, \widetilde{\alpha}),(\widetilde{\alpha}, \widetilde{s})$ for fermions). Appendix A gives a detailed description of the construction of the twist operators and computations using them. For our immediate purposes, the relevant point is that the integers

$$
\left\{N_{n \mu}, N_{n \mu}^{\prime}\right\}
$$

uniquely specify a Ramond ground state.

\subsection{Map to the FP system and microstate geometries}

It has been proposed that each Ramond ground state of the D1-D5 has a corresponding exact spacetime geometry without horizons [22]. The construction of these geometries was carried out by first $U$-dualizing the D1-D5 system to the FP system in type II, where an F1 string is wound $N_{5}$ times along $S^{1}$ and carries $N_{1}$ units of momentum in the $S^{1}$ direction [44]. If the right-moving oscillation number $N_{R}$ vanishes, $N_{R}=0$, then this configuration is BPS. Such states can be written as

$$
\begin{aligned}
& \prod_{n, \mu}\left(\alpha_{-n}^{\mu}\right)^{N_{n \mu}}\left(\psi_{-n}^{\mu}\right)^{N_{n \mu}^{\prime}}\left|N_{1}, N_{5}\right\rangle \\
& N_{L}=\sum_{n, \mu} n\left(N_{n \mu}+N_{n \mu}^{\prime}\right)=N_{1} N_{5}=N, \quad N_{n \mu}=0,1,2, \ldots, \quad N_{n \mu}^{\prime}=0,1 .
\end{aligned}
$$


Here $\alpha_{-n}^{\mu}$ and $\psi_{-n}^{\mu}$ are left-moving bosonic and fermionic oscillators, respectively. The polarization $\mu$ runs over eight transverse directions. $\left|N_{1}, N_{5}\right\rangle$ is the F1 string state with momentum $N_{1}$ and winding number $N_{5}$, and with no oscillators excited (this state itself is not physical). The second line displays the Virasoro constraint on the left-moving oscillation number $N_{L}$.

Following [22, 36], the $U$-duality map between the states $(2.5)$ of the FP system and the Ramond ground states (2.3) of the D1-D5 system is given by

$$
\sigma_{n}^{\mu} \leftrightarrow \alpha_{-n}^{\mu} \quad \tau_{n}^{\mu} \leftrightarrow \psi_{-n}^{\mu} .
$$

The set of integers (2.4) defining a Ramond ground state is precisely mapped into the set of integers defining an excitation of the FP system.

The metric of the FP system is known for arbitrary classical profile $x^{\mu}=F^{\mu}(v)$ of the F1 string by the chiral null model [45-48]. Here $\mu$ runs over the eight transverse directions to the F1 worldsheet. $v=t-y$ is the left-moving lightcone coordinate, reflecting the fact that there must be only left-moving waves on the F1 string because of the BPS condition. By $U$-dualizing back, Lunin and Mathur [22] obtained the metric of the D1-D5 system, when the classical profile $F^{\mu}(v)$ is only in the noncompact $\mathbb{R}^{4}$ directions $\mathbf{x}=x^{i}, i=1,2,3,4$. Explicitly, the string frame metric of the D1-D5 system in the decoupling limit is given by $[22,36]$

$\mathrm{d} s_{\text {string }}^{2}=\frac{1}{\sqrt{f_{1} f_{5}}}\left[-(\mathrm{d} t-A)^{2}+(\mathrm{d} y+B)^{2}\right]+\sqrt{f_{1} f_{5}} \mathrm{~d} x^{i} \mathrm{~d} x^{i}+\sqrt{\frac{f_{1}}{f_{5}}} \mathrm{~d} z^{a} \mathrm{~d} z^{a}$,

$\mathrm{e}^{2 \Phi}=\frac{f_{1}}{f_{5}}, \quad f_{5}=\frac{Q_{5}}{L} \int_{0}^{L} \frac{\mathrm{d} v}{|\mathbf{x}-\mathbf{F}(v)|^{2}}, \quad f_{1}=\frac{Q_{5}}{L} \int_{0}^{L} \frac{|\dot{\mathbf{F}}(v)|^{2} \mathrm{~d} v}{|\mathbf{x}-\mathbf{F}(v)|^{2}}$,

$A_{i}=-\frac{Q_{5}}{L} \int_{0}^{L} \frac{\dot{F}_{i}(v) \mathrm{d} v}{|\mathbf{x}-\mathbf{F}(v)|^{2}}, \quad \mathrm{~d} B=-*_{4} \mathrm{~d} A$.

Here, $y$ and $z^{a}$ are $S^{1}$ and $T^{4}$ directions, respectively. The coordinate radius of $S^{1}$ is $R$, and the coordinate volume of $T^{4}$ is $(2 \pi)^{4} V_{4}$. The length $L$ is related to $R$ by

$$
L=\frac{2 \pi g_{s} \alpha^{\prime} N_{5}}{R}=\frac{2 \pi Q_{5}}{R},
$$

where the D5-brane charge $Q_{5}$ is related to the D5 number $N_{5}$ by $Q_{5}=g_{s} \alpha^{\prime} N_{5}$. The four arbitrary functions $\mathbf{F}(v)=F_{i}(v), 0 \leqslant v \leqslant L$ in (2.8) parametrize the solution, and correspond to the classical profile $\mathbf{F}_{\mathrm{FP}}(v)$ of the $\mathrm{F} 1$ string in the FP duality frame by $\mathbf{F}(v)=\mu \mathbf{F}_{\mathrm{FP}}(v), \mu=g_{s} \alpha^{\prime 3 / 2} / R \sqrt{V_{4}}$ [22]. The D1 charge is given by

$$
Q_{1}=\frac{Q_{5}}{L} \int_{0}^{L}|\dot{\mathbf{F}}(v)|^{2} \mathrm{~d} v
$$

The D1-brane charge $Q_{1}$ is related to the D1 number $N_{1}$ by $Q_{1}=g_{s} \alpha^{\prime 3} N_{1} / V_{4}$. In this paper, we will argue that typical probes of typical microstate geometries will react as if the spacetime was simply an $M=0$ BTZ black hole (2.13) below.

Using (2.6) the Ramond ground states can be mapped onto specific states of the FP system (2.5). For states involving only $\alpha_{-n}^{i}$ this in turn determines the classical profile $F^{i}(v)$ of the F1 string, which can be substituted into (2.8) to give the proposed geometry corresponding to a specific Ramond ground state. Details and examples are given in [22]. For example, the special Ramond ground state $\left[\sigma_{n}^{s \tilde{s}}\right]^{N / n}$ with $s=\tilde{s}=-1,1 \leqslant n \leqslant N$ corresponds to the bulk geometry $\left(\operatorname{AdS}_{3} \times S^{3}\right) / \mathbb{Z}_{n} \times T^{4}$. The three-dimensional part of this geometry is the conical defect described below. For general states involving all bosonic oscillators as well as fermionic oscillators more work is needed; see [29] for results regarding the fermionic states. 


\subsection{Bulk geometries and correlators}

In this subsection, we will review some geometries that show up as the bulk geometries in the context of AdS/CFT for the D1-D5 system. We will also present the bulk two-point functions of a massless minimally coupled scalar in those geometries, and compare them at the end. The asymptotic $\mathrm{AdS}_{3}$ radius is given by $\ell \sim N^{1 / 4}$.

2.3.1. Conical defect. The Ramond ground state $\left[\sigma_{n}^{s \tilde{s}}\right]^{N / n}$ with $s=\tilde{s}=-1,1 \leqslant n \leqslant N$ corresponds in the bulk to the conical defect geometry $[37,38]$ :

$$
\mathrm{d} s^{2}=-\left(\frac{1}{n^{2}}+\frac{r^{2}}{\ell^{2}}\right) \mathrm{d} t^{2}+\frac{\mathrm{d} r^{2}}{\frac{1}{n^{2}}+\frac{r^{2}}{\ell^{2}}}+r^{2} \mathrm{~d} \phi^{2}
$$

Here $n$ is an integer in the range $1 \leqslant n \leqslant N$. The angular identification is $(\phi, \psi) \cong$ $\left(\phi+2 \pi, \psi+\frac{2 \pi}{n}\right)$, where $\psi$ is an angle on the $S^{3}$ factor that we have suppressed. The special case $n=1$ yields $\mathrm{AdS}_{3}$ in global coordinates.

Any state can be probed by computing the correlation functions of operators in that state. The simplest correlator that one could compute, the two-point function, is related to a fourpoint function computed in the vacuum. According to the AdS/CFT correspondence, the two-point function in the state $\left[\sigma_{n}^{s \tilde{s}}\right]^{N / n}, s=\widetilde{s}=-1$ can be obtained from AdS space by computing the bulk-boundary propagator of the spacetime field that is dual to the CFT probe and then taking the bulk point to the boundary. Let us consider a CFT probe that is dual to a massless scalar field in $\mathrm{AdS}_{3}$. The conical defect propagator for this field is obtained from the AdS propagator by summing over the images that define the conical defect. By translation invariance we can take one of the boundary points to be at $t=\phi=0$. We then obtain the result

$$
\begin{aligned}
& \sum_{k=0}^{n-1} \frac{1}{\left(2 n \sin \frac{w-2 \pi k}{2 n}\right)^{2}\left(2 n \sin \frac{\bar{w}-2 \pi k}{2 n}\right)^{2}} \\
& =\frac{1}{16 n^{2} \sin ^{2} \frac{w-\bar{w}}{2 n}}\left[\frac{1}{\sin ^{2} \frac{w}{2}}+\frac{1}{\sin ^{2} \frac{\bar{w}}{2}}-\frac{2 \sin \frac{w-\bar{w}}{2}}{n \tan \frac{w-\bar{w}}{2 n} \sin \frac{w}{2} \sin \frac{\bar{w}}{2}}\right]
\end{aligned}
$$

where

$$
w=\phi-\frac{t}{\ell}, \quad \bar{w}=\phi+\frac{t}{\ell} .
$$

The summation was done by a standard contour integration method.

2.3.2. Naive geometry. Consider taking the $n \rightarrow \infty$ limit of the conical defect geometries:

$$
\mathrm{d} s^{2}=-\frac{r^{2}}{\ell^{2}} \mathrm{~d} t^{2}+\frac{\ell^{2}}{r^{2}} \mathrm{~d} r^{2}+r^{2} \mathrm{~d} \phi^{2}
$$

This is the same as $\mathrm{AdS}_{3}$ in Poincaré coordinates with a periodically identified spatial direction. This geometry does not actually correspond to any CFT microstate since it has $n>N$. Instead, we will see that this geometry emerges as an effective description of the typical Ramond ground state at large $N$. As before we compute the boundary two-point function for a massless scalar field, and find that

$$
\sum_{k=-\infty}^{\infty} \frac{1}{(w-2 \pi k)^{2}(\bar{w}-2 \pi k)^{2}}=\frac{1}{4(w-\bar{w})^{2}}\left[\frac{1}{\sin ^{2} \frac{w}{2}}+\frac{1}{\sin ^{2} \frac{\bar{w}}{2}}-\frac{4 \sin \frac{w-\bar{w}}{2}}{(w-\bar{w}) \sin \frac{w}{2} \sin \frac{\bar{w}}{2}}\right] .
$$


2.3.3. Non-rotating BTZ. The above naive geometry is in fact the massless limit of the BTZ black holes of $\mathrm{AdS}_{3}$. To see this, recall that the non-rotating BTZ black holes have a metric [30]

$$
\mathrm{d} s^{2}=-\frac{r^{2}-r_{+}^{2}}{\ell^{2}} \mathrm{~d} t^{2}+\frac{\ell^{2}}{r^{2}-r_{+}^{2}} \mathrm{~d} r^{2}+r^{2} \mathrm{~d} \phi^{2}
$$

If we take $r_{+}=0$, which is the $M=0$ BTZ black hole, we get back the naive geometry (2.13). The boundary two-point function is [49]

$$
\frac{16 r_{+}^{4}}{4 \ell^{4}} \sum_{k=-\infty}^{\infty}\left[\frac{1}{\sinh \left(\frac{r_{+}}{2 \ell}(w+2 \pi k)\right) \sinh \left(\frac{r_{+}}{2 \ell}(\bar{w}+2 \pi k)\right)}\right]^{2} .
$$

We have not succeeded in doing the summation in closed form. But we can use contour integration to rewrite the sum in a way which makes the large time behaviour manifest. For simplicity set $\phi=0$. Then one can rewrite (2.16) as

$$
\begin{aligned}
\frac{r_{+}^{2}}{8 \ell^{2}} \frac{1}{\sinh ^{2}\left(\frac{r_{+} t}{\ell^{2}}\right)} & \left\{\sum_{m=-\infty}^{\infty}\left[\frac{1}{\sin ^{2}\left(\frac{t}{2 \ell}+\mathrm{i} \pi \frac{\ell}{r_{+}} m\right)}+\frac{2 r_{+}}{\ell} \frac{1}{\tan \left(\frac{t}{2 \ell}+\mathrm{i} \pi \frac{\ell}{r_{+}} m\right) \tanh \left(\frac{r_{+} t}{\ell^{2}}\right)}\right]\right. \\
& \left.+\frac{2 r_{+}}{\pi \ell}\left[\frac{\left(\frac{r_{+} t}{\ell^{2}}\right)}{\tanh \left(\frac{r_{+} t}{\ell^{2}}\right)}-1\right]\right\} .
\end{aligned}
$$

To simplify further, consider the case of a small black hole, $r_{+} \ll \ell$. In this case, we can truncate to just the $m=0$ term and obtain

$\frac{r_{+}^{2}}{8 \ell^{2}} \frac{1}{\sinh ^{2}\left(\frac{r_{+} t}{\ell^{2}}\right)}\left\{\frac{1}{\sin ^{2}\left(\frac{t}{2 \ell}\right)}+\frac{2 r_{+}}{\ell} \frac{1}{\tan \left(\frac{t}{2 \ell}\right) \tanh \left(\frac{r_{+} t}{\ell^{2}}\right)}+\frac{2 r_{+}}{\pi \ell}\left[\frac{\left(\frac{r_{+} t}{\ell^{2}}\right)}{\tanh \left(\frac{r_{+} t}{\ell^{2}}\right)}-1\right]\right\}$.

2.3.4. Comparison. Note that the three geometries described above look the same outside a core region. As we will review later, the $n$ appearing in the conical defect geometry has a typical size

$$
n_{\text {typ }} \sim N^{1 / 2} \sim \ell^{2}
$$

The typical conical defect geometry thus approaches the naive geometry for $r \gg \ell^{-1}$. Consider then the BTZ geometry with $r_{+}=\ell^{-1}$, so that it has the same characteristic size as the typical conical defect. The Bekenstein-Hawking entropy of this black hole is then

$$
S \sim A \sim \ell^{3} r_{+} \sim \ell^{2} \sim N^{1 / 2}
$$

where the factor of $\ell^{3}$ came from integration over the $S^{3}$ that we have suppressed. $S \sim N^{1 / 2}$ is indeed the correct ground state entropy of the D1-D5 system. This is an example of the stretched horizon idea advocated in [22].

The most obvious difference between the two-point functions computed above is that the conical defect result is periodic in time, with a period $\Delta t=2 \pi n \ell$, while the naive geometry and the BTZ black hole results decay to zero at large time. Usually, this sort of decay is associated with the presence of a horizon, with the information loss problem arising because the decay winds up implying a failure of unitarity [4]. Later in this paper we will show that the decay is the correct universal description of the typical two-point function in a typical state, but that its persistence to late times is an artefact of ignoring the precise quantum mechanical details of the individual microstates of a black hole. Our computations will be for the $M=0$ BTZ black hole which will turn out to be the effective coarse-grained description of the typical Ramond ground state of the dual CFT. To this end, we now turn to characterizing the structure of these states. 


\section{Typical states}

\subsection{Statistics and typical states}

As described in section 2.1, each ground state in the Ramond sector of the D1-D5 CFT is characterized by a set of integers $\left\{N_{n \mu}, N_{n \mu}^{\prime}\right\}$ specifying the distribution of constituent bosonic and fermionic twists (2.3). When the total twist length $N=\sum_{n, \mu} n\left(N_{n \mu}+N_{n \mu}^{\prime}\right)$ is very large, there is a macroscopic number $\left(\sim \mathrm{e}^{2 \sqrt{2} \pi \sqrt{N}}\right)$ of Ramond ground states. In such a situation, most of those $\mathrm{e}^{2 \sqrt{2} \pi \sqrt{N}}$ microstates will have a twist distribution $\left\{N_{n \mu}, N_{n \mu}^{\prime}\right\}$ that lies very close to a certain 'typical' distribution. In the large $N$ limit, the difference among individual distributions is small. Roughly, statistical mechanics states that $\left\langle\left(\Delta N_{n \mu}\right)^{2}\right\rangle \sim N_{n \mu}$, thus $\frac{\left\langle\left(\Delta N_{n \mu}\right)^{2}\right\rangle^{1 / 2}}{N_{n \mu}} \sim\left(N_{n \mu}\right)^{-1 / 2} \rightarrow 0$ as $N_{n \mu} \rightarrow \infty$. Thus, although correlation functions computed in individual microstates depend on the precise form of the microstate distribution $\left\{N_{n \mu}, N_{n \mu}^{\prime}\right\}$, for almost all microstates the generic responses should deviate by small amounts from the results for the typical state. In the next section, this will be the basis for the emergence of an effective black hole description of typical Ramond ground states. A similar analysis of the typical states was carried out for the $\mathrm{AdS}_{5} / \mathrm{SYM}_{4}$ duality in [34].

In this section, our goal is to characterize the typical distribution of twists and the size of fluctuations around it within the ensemble of Ramond ground states. Ideally, we carry out a microcanonical analysis by studying all partitions of integers (2.3) that lead to a total twist of $N$. However, it is easier to carry out a canonical analysis by including states with an arbitrary total twist into the ensemble, while fixing the average total twist to be $N$ via an effective temperature $T$. The relative error incurred by the canonical approach compared to the exact microcanonical analysis vanishes in the large $N$ limit. Large $N$ will correspond to high temperature $T \gg 1$, or equivalently, small $\beta=1 / T \ll 1$. Since the constituent twist operators in (2.3) carry an $R$-charge, we can study the structure of Ramond ground states restricted to carry some fixed $R$-charge. In particular, in terms of the $S U(2)_{R} \times \widetilde{S U(2)}_{R}$ charges in (2.2), let us define

$$
J=-J_{R}^{3}-\widetilde{J}_{R}^{3}, \quad \tilde{J}=J_{R}^{3}-\widetilde{J}_{R}^{3} .
$$

With these definitions, the $R$-charges $J$ and $\tilde{J}$ correspond in the bulk geometry to orthogonal angular momenta in the $\mathbb{R}^{4}$ perpendicular to the D1-D5 worldvolume ${ }^{5}$. We will consider the structure of Ramond ground states with $\tilde{J}=0$ and different values of $J$.

\subsection{Typical twist distribution with $J=\widetilde{J}=0$}

Let us first consider the ensemble of all the Ramond ground states (2.3) with equal statistical weight. Some of the states in this ensemble will have a non-vanishing $R$-charge. However, because the polarizations $\mu$ of twist operators $\sigma_{n}^{\mu}, \tau_{n}^{\mu}$ are weighted equally, on average the states will have $J=\tilde{J}=0$. Indeed, as we will see, there are so many more states with $J=0$ than $J \neq 0$ that summing over all states only incurs a small error in studying the properties of $J=0$ states.

As described in (2.3) and appendix A, we have eight bosonic twist operators $\sigma_{n}^{\mu}$ and eight fermionic twist operators $\tau_{n}^{\mu}$ which are all independent. So the canonical partition function is

$Z(\beta)=\operatorname{Tr}\left[\mathrm{e}^{-\beta N}\right]=\prod_{n=1}^{\infty} \frac{\left(1+q^{n}\right)^{8}}{\left(1-q^{n}\right)^{8}}=\left[\frac{\vartheta_{2}(0 \mid \tau)}{2 \eta(\tau)^{3}}\right]^{4}, \quad q=\mathrm{e}^{2 \pi \mathrm{i} \tau}=\mathrm{e}^{-\beta}$.

5 If we let $\mathbb{R}^{4}$ coordinates be $x^{1,2,3,4}$, the two angular momenta in question are $J_{12}$ and $J_{34}$. See appendix A.1 for details. 
Using the modular property of the theta function,

$$
Z(\beta)=\left[\frac{\beta}{4 \pi} \frac{\vartheta_{4}\left(0 \mid-\frac{1}{\tau}\right)}{\eta\left(-\frac{1}{\tau}\right)^{3}}\right]^{4} \sim \mathrm{e}^{2 \pi^{2} / \beta} \quad(\beta \ll 1) .
$$

The relation between 'energy' $N$ and temperature $\beta$ is

$$
N=\left\langle\sum_{n=1}^{\infty} \sum_{\mu} n\left(N_{n \mu}+N_{n \mu}^{\prime}\right)\right\rangle=-\frac{\partial}{\partial \beta} \ln Z(\beta) \simeq \frac{2 \pi^{2}}{\beta^{2}}
$$

Since all twist operators are independent, the average distribution $\left\{N_{n \mu}, N_{n \mu}^{\prime}\right\}$ is given by the Bose-Einstein and Fermi-Dirac distribution, respectively:

$$
N_{n \mu}=\frac{1}{\mathrm{e}^{\beta n}-1}, \quad N_{n \mu}^{\prime}=\frac{1}{\mathrm{e}^{\beta n}+1}, \quad N_{n}=\sum_{\mu}\left(N_{n \mu}+N_{n \mu}^{\prime}\right)=\frac{8}{\sinh \beta n} .
$$

For large $N$, the typical states of our ensemble have a distribution almost identical to (3.5). We will call the distribution (3.5) the 'representative' distribution.

\subsection{Typical twist distribution with $J \neq 0$ and $\tilde{J}=0$}

Now let us consider the typical state in the ensemble with fixed $R$-charge $J \neq 0$. From the definitions in section 2.1 , the twist operators that carry nonzero $J$ are

$$
\sigma_{n}^{s \tilde{s}}: J=-(s+\tilde{s}) / 2, \quad \tau_{n}^{s \widetilde{\alpha}}: J=-s / 2, \quad \tau_{n}^{\widetilde{\alpha s}}: J=-\widetilde{s} / 2
$$

Strictly speaking, we should consider the microcanonical ensemble in which $N$ and total $J$ are fixed. But again in the large $N$ limit, we can equivalently consider the canonical ensemble in which $N$ and $J$ are controlled by temperature $\beta$ and chemical potential $\mu$.

To construct the partition function it is convenient to use the map (2.6) between the Ramond ground states and the FP system. Then we are equivalently constructing the ensemble left-moving oscillations of the FP string as specified in (2.5). In the FP language, we consider an ensemble in which we have $N_{B}$ left-moving bosons $\alpha_{-n}^{i}$ and $N_{F}$ left-moving fermions $\psi_{-n}^{i}$, where $n=1,2, \ldots$ Let the bosons and fermions carry the following $R$-charge assignments:

$\begin{array}{llllll}n_{B} & \text { bosons: } & J=+1 & n_{F} & \text { fermions: } & J=+1 / 2 \\ n_{B} & \text { bosons: } & J=-1 & n_{F} & \text { fermions: } & J=-1 / 2 \\ N_{B}-2 n_{B} & \text { bosons: } & J=0 & N_{F}-2 n_{F} & \text { fermions: } & J=0\end{array}$

The case we are interested in, i.e., the D1-D5 system on $T^{4}$, has $N_{B}=N_{F}=8, n_{B}=1$, $n_{F}=4$. The D1-D5 system on $\mathrm{K} 3$ has $N_{B}=24, n_{B}=1, N_{F}=n_{F}=0$, for which state counting was first studied in [50] from the heterotic dual perspective. More recently, the microscopics of the $\mathrm{K} 3$ case was studied in [21], and the discussions below and in appendix B are generalization of the one therein.

We can compute the entropy $S(N, J)$ for given level $N$ and $R$-charge $J$ by studying the partition function

$$
\begin{aligned}
Z(\beta, \mu) & =\sum_{N, J} \mathrm{~d}_{N, J} q^{N} z^{J}=\operatorname{Tr}\left[\mathrm{e}^{-\beta(N-\mu J)}\right] \\
& =\prod_{n=1}^{\infty} \frac{\left[\left(1+z^{1 / 2} q^{n}\right)\left(1+z^{-1 / 2} q^{n}\right)\right]^{n_{F}}\left(1+q^{n}\right)^{N_{F}-2 n_{F}}}{\left[\left(1-z q^{n}\right)\left(1-z^{-1} q^{n}\right)\right]^{n_{B}}\left(1-q^{n}\right)^{N_{B}-2 n_{B}}}
\end{aligned}
$$


where $q=\mathrm{e}^{2 \pi \mathrm{i} \tau}=\mathrm{e}^{-\beta}, z=\mathrm{e}^{2 \pi \mathrm{i} v}=\mathrm{e}^{\beta \mu}$. The entropy in the $N \rightarrow \infty$ limit can be evaluated by thermodynamic approximation, as explained in appendix $\mathrm{B}$, and the result is

$$
S=\log \mathrm{d}_{N, J}=2 \pi \sqrt{\frac{c}{6}(N-|J|)} .
$$

Now let us apply this to the D1-D5 system on $T^{4}$, for which $N_{B}=N_{F}=8, n_{B}=1$, $n_{F}=4$. One sees from (3.7) that the only effect of $J \neq 0$ is to replace $N$ with $\widetilde{N} \equiv N-J$. Here we assumed $J>0$. This means that almost all states (i.e., the states that are responsible for the entropy) in the ensemble with level $N$ and $R$-charge $J$ are of the form

$$
\left(\alpha_{-1}^{+}\right)^{J} \underbrace{\prod_{n=1}^{\infty}\left[\prod_{i}\left(\alpha_{-n}^{i}\right)^{N_{n i}}\left(\psi_{-n}^{i}\right)^{N_{n i}^{\prime}}\right]|0\rangle}_{\begin{array}{l}
\text { states that are responsible for entropy of the } \\
\text { ensemble with level } \widetilde{N} \\
\text { angular momentum }
\end{array}}
$$

where $\alpha_{-n}^{+}{ }^{\dagger}$ is the creation operator of the boson that carries $J=+1$. Indeed, the entropy from the ' $\underbrace{\ldots}$ ' part is $2 \pi \sqrt{c \widetilde{N} / 6}=2 \pi \sqrt{c(N-J) / 6}$, which fully accounts for (3.7). Of course, besides $\left(\alpha_{-1}^{+}\right)^{J}$ there are other combinations of oscillators that can carry $R$-charge $J$. But any other combination will exact more price in $N$, and will therefore lead to a subleading contribution to the entropy. If $J<0$, then the same argument goes through if we replace $\left(\alpha_{-1}^{+}\right)^{J}$ with $\left(\alpha_{-1}^{-\dagger}\right)^{|J|}$.

Translating the above into the language of the D1-D5 system, the typical state of the D1-D5 system with $N \gg 1$ and $J \neq 0$ splits into the following two parts:

(1) $|J|$ strings of unit length, $\left(\sigma_{1}^{s \widetilde{s}}\right)^{|J|}$, where $s=\tilde{s}=-1$ for $J>0$ and $s=\widetilde{s}=+1$ for $J<0$. We will call this part the 'Bose-Einstein (BE) condensate'.

(2) The typical state of the ensemble with $\sum_{n \mu} n\left(N_{n \mu}+N_{n \mu}^{\prime}\right)=\tilde{\tilde{N}}=N-|J|$ and no $R$-charge.

In other words, the typical distribution $\left\{N_{n \mu}, N_{n \mu}^{\prime}\right\}$ of the ensemble with level $N$ and angular momentum $J$ can be written as

$$
N_{n \mu}=N_{n \mu}^{(\mathrm{BEC})}+\tilde{N}_{n \mu}, \quad N_{n \mu}^{\prime}=\tilde{N}_{n \mu}^{\prime},
$$

where the BE condensate part $N_{n \mu}^{(\mathrm{BEC})}$ is given by

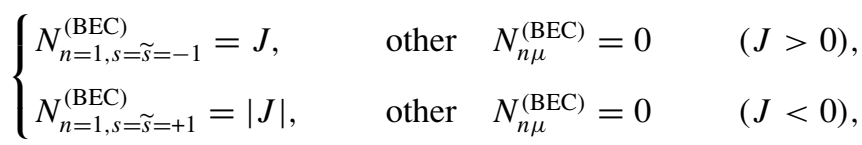

while the non-condensate part $\left\{\widetilde{N}_{n \mu}, \widetilde{N}_{n \mu}^{\prime}\right\}$ is identical to the typical distribution (3.5) for the ensemble with level $\widetilde{N}=N-|J|$ and no $R$-charge.

Note that the entropy of the ensemble with $J=0$ is the same as that in the ensemble with $J$ unspecified. This is a reflection of the fact that there are exponentially more states with $J=0$ than with $J \neq 0$.

\section{The effective geometry}

In the previous section, we derived the distributions of constituent twist operators in typical Ramond ground states with $R$-charges $J=0$ and $J \neq 0$. In the large $N$ limit, almost all states with the given charges have twist distributions that lie close to these typical distributions. 
Here we will compute two-point correlation functions in typical states and show that generic correlators computed at finite-time separations are largely independent of the details of the microstate. Indeed, at small time separations two-point correlators in the typical $J=0$ state give a universal response, as if the corresponding spacetime geometry was a black hole. By contrast, atypical correlators whose two insertion points are separated by very long times give responses with intricate variations that encode the detailed microstate.

The fact that generic probes of typical states give essentially universal responses governed by the statistics of the state's microscopic constituents is reminiscent of the similar observation in [34] for the case of $\mathrm{AdS}_{5} / \mathrm{SYM}_{4}$. There, it was argued that the correlation function of a small 'probe' operator $\mathcal{A}$ in a black hole background produced by a long operator $\mathcal{O}$ is determined by the matching of patterns of fields $(X, Y, Z, \bar{X}$, etc) in both operators, the distribution of which is governed largely by statistics. In the case of half-BPS states of the $\mathrm{AdS}_{5} / \mathrm{SYM}_{4}$ theory [31-33] it was possible to use the Yang-Mills theory to argue for an effective spacetime description of microstates in terms of a singular geometry [34]. In the D1-D5 case, we have already noted that there exists a proposed map taking RR ground states into geometries, and we argue below that this map will also give an effective singular spacetime description to typical states. However, to genuinely prove these assertions it is necessary to compute correlation functions, because it is through correlators that we can rigorously compare bulk and boundary physics in the AdS/CFT correspondence. Here we will carry out the analysis of deriving an effective geometry from correlation functions and thereby infer the effective geometry corresponding to a typical state. Furthermore, the maps between states and geometries, either based on LLM [33] or on the Lunin-Mathur geometries, are only valid at the level of twoderivative supergravity. To learn anything about the geometry when this approximation breaks down it is necessary to extract the spacetime physics from CFT correlation functions (or augment the original map with higher derivative terms). We will give an explicit example of this here. At late times our correlators probe the strongly curved region of the geometry where the effective spacetime description breaks down. The CFT correlators continue to be valid and give a result that depends on which particular microstate one has chosen. This shows how the CFT can be used to go beyond the accuracy of the low energy spacetime description.

\subsection{Two-point functions of the D1-D5 CFT}

For simplicity, we will compute the two-point functions of non-twist 'probe' operators $\mathcal{A}$ in states created by general twist operators. $\mathcal{A}$ can be written as a sum over copies of the CFT,

$$
\mathcal{A}=\frac{1}{\sqrt{N}} \sum_{A=1}^{N} \mathcal{A}_{A}
$$

where $\mathcal{A}_{A}$ is a non-twist operator that lives in the $A$ th copy. For example, we can take

$$
\mathcal{A}_{A}=\partial X_{A}^{a}(z) \bar{\partial} X_{A}^{b}(\bar{z})
$$

which corresponds to a fluctuation of the metric in the internal $T^{4}$ direction. Although, such non-twist operators are only a subset of the operators that correspond to spacetime excitations, we will restrict ourselves to them because their correlation functions are much easier to compute than those of twist operators, and because they will be sufficient to demonstrate that an effective geometry emerges in the $N \rightarrow \infty$ limit.

Given a general Ramond ground state $\sigma$ (2.3) we are interested in computing

$$
\left\langle\sigma^{\dagger} \mathcal{A}^{\dagger} \mathcal{A} \sigma\right\rangle \text {. }
$$

The key result, demonstrated in appendix A, is that for non-twist operators at the orbifold point in the CFT such correlation functions decompose into independent contributions from 
the constituent twists operators in (2.3). Denoting the constituents $\sigma_{n}^{\mu}, \tau_{n}^{\mu}$ collectively by $\sigma_{n}^{\hat{\mu}}$ and $N_{n \mu}, N_{n \mu}^{\prime}$ by $N_{n \hat{\mu}}$, we write the Ramond ground states (2.3) as

$$
\sigma=\prod_{n, \hat{\mu}}\left(\sigma_{n}^{\hat{\mu}}\right)^{N_{n \hat{\mu}}}
$$

Then, the desired correlation function decomposes as

$$
\left\langle\sigma^{\dagger} \mathcal{A}^{\dagger} \mathcal{A} \sigma\right\rangle=\frac{1}{N} \sum_{n, \hat{\mu}} n N_{n \hat{\mu}} \sum_{A=1}^{n}\left\langle\left[\sigma_{n}^{\hat{\mu}}\right]^{\dagger} \mathcal{A}_{A}^{\dagger} \mathcal{A}_{1} \sigma_{n}^{\hat{\mu}}\right\rangle .
$$

The problem then reduces to computing four-point functions of the form

$\left\langle\left[\sigma_{(1, \ldots, n)}^{\widehat{\mu}}(z=\infty)\right]^{\dagger} \mathcal{A}_{A}\left(z_{1}\right)^{\dagger} \mathcal{A}_{B}\left(z_{2}\right) \sigma_{(1, \ldots, n)}^{\widehat{\mu}}(z=0)\right\rangle \equiv\left\langle\mathcal{A}_{A}\left(z_{1}\right)^{\dagger} \mathcal{A}_{B}\left(z_{2}\right)\right\rangle_{\sigma_{(1, \ldots, n)}^{\widehat{\mu}}}$,

where $1 \leqslant A, B \leqslant n$, and the equation indicates that we are equivalently computing the two-point function of $\mathcal{A}$ in the ground state of twist sector $n$. As we described, in the $n$th twist sector the worldsheet is effectively $n$ times as long and therefore, as shown in appendix A, for bosonic operators

$$
\left\langle\mathcal{A}_{A}^{\dagger}\left(w_{1}\right) \mathcal{A}_{B}\left(w_{2}\right)\right\rangle_{\sigma_{(1, \ldots, n)}}=\frac{C}{\left[2 n \sin \left(\frac{w}{2 n}\right)\right]^{2 h}\left[2 n \sin \left(\frac{\bar{w}}{2 n}\right)\right]^{2 \tilde{h}}},
$$

where

$$
w \equiv w_{1}-w_{2}, \quad \bar{w} \equiv \bar{w}_{1}-\bar{w}_{2} .
$$

Here, the copy labels $A, B$ mean that $w_{1}$ and $w_{2}$ must be understood as $w_{1}+2 \pi(A-1)$ and $w_{2}+2 \pi(B-1)$, respectively. The analogous computation for fermionic $\mathcal{A}$ is given in appendix A.6.

\subsection{Example of typical state correlation function}

The correlation function of non-twist operators in the general microstate (2.3) can be computed by plugging (4.7) and (A.40) into the general formula (4.5). For example, for $\mathcal{A}$ purely bosonic, we substitute bosonic correlator (4.7) into (4.5) to obtain

$$
\left\langle\mathcal{A}\left(w_{1}\right) \mathcal{A}\left(w_{2}\right)\right\rangle_{\Sigma}=\frac{1}{N} \sum_{n} n N_{n} \sum_{k=0}^{n-1} \frac{C}{\left[2 n \sin \left(\frac{w-2 \pi k}{2 n}\right)\right]^{2 h}\left[2 n \sin \left(\frac{\bar{w}-2 \pi k}{2 n}\right)\right]^{2 \tilde{h}}},
$$

where

$$
N_{n} \equiv \sum_{\mu}\left(N_{n \mu}+N_{n \mu}^{\prime}\right)
$$

Here we took into account that the copy labels $A, B$ mean that $w$ in (4.7) should be replaced by $w+2 \pi(A-B)$. The correlation function for the typical state is obtained simply by plugging the typical distribution (3.5) or (3.9) into $\left\{N_{n \mu}, N_{n \mu}^{\prime}\right\}$ above. For fermionic $\mathcal{A}$, the correlation function (A.29) depends on the spin $\mu$ and the expression is more complicated, as we will see below.

As a simple example of a probe $\mathcal{A}$, take the operator (4.2) which is dual to a fluctuation of the metric on $T^{4}$. For this operator $h=\tilde{h}=1$. In this case, the summation over $k$ in (4.9) is the same as in (2.11). Therefore the correlation function can be written as

$$
\begin{aligned}
G(w, \bar{w}) & \equiv\left\langle\partial X \bar{\partial} X\left(w_{1}\right) \partial X \bar{\partial} X\left(w_{2}\right)\right\rangle_{\Sigma} \\
& =-\frac{1}{N} \sum_{n=1}^{\infty} \frac{n N_{n}}{16 n^{2} \sin ^{2} \frac{w-\bar{w}}{2 n}}\left[\frac{1}{\sin ^{2} \frac{w}{2}}+\frac{1}{\sin ^{2} \frac{\bar{w}}{2}}-\frac{2 \sin \frac{w-\bar{w}}{2}}{n \tan \frac{w-\bar{w}}{2 n} \sin \frac{w}{2} \sin \frac{\bar{w}}{2}}\right] .
\end{aligned}
$$



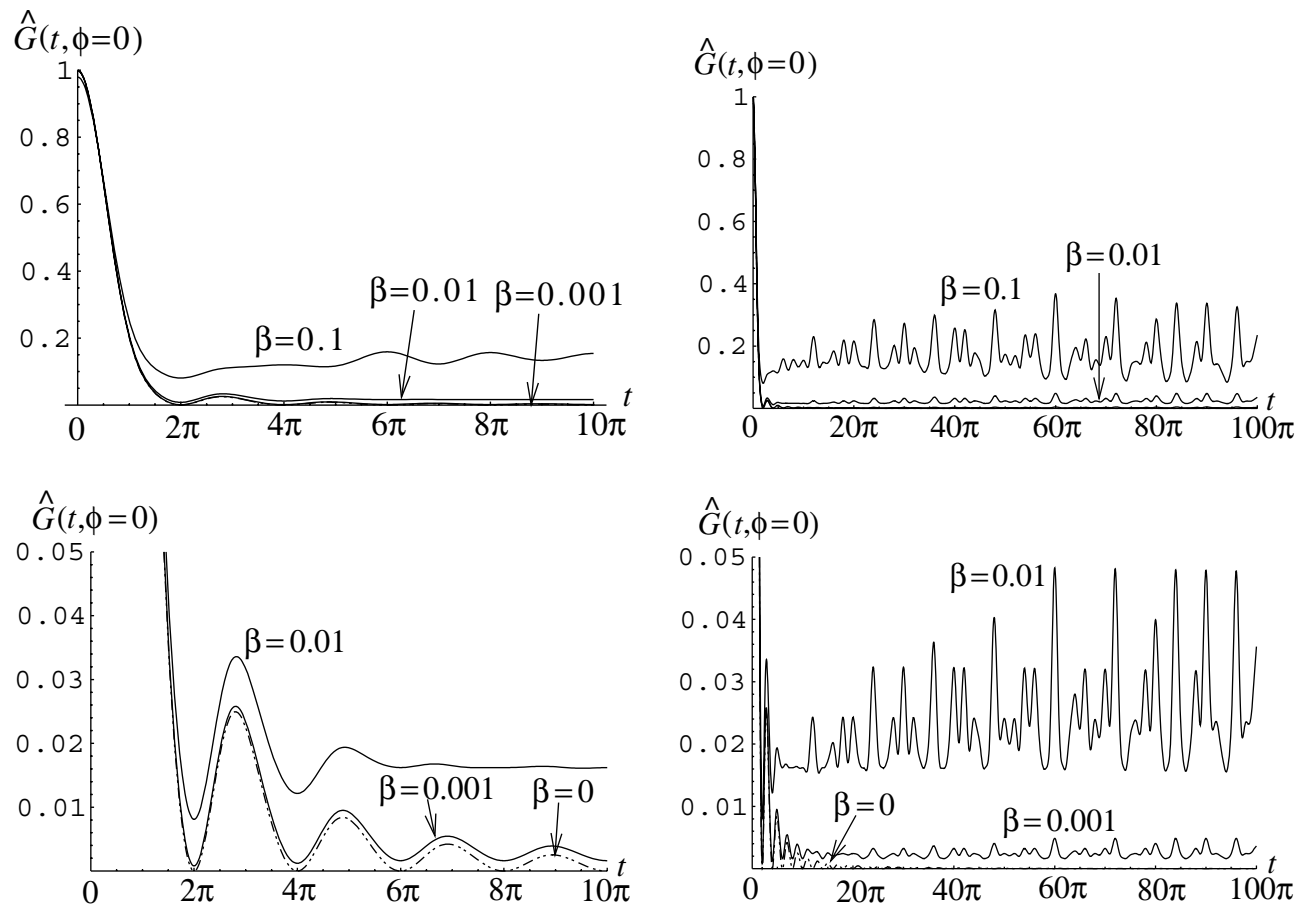

Figure 1. Plot of the regularized correlation function $\widehat{G}(t, \phi=0 ; \beta)$ as a function of $t$, for various values of $\beta$. The two graphs on the left show short-time behaviour; the two on the right show long-time behaviour. As $\beta \rightarrow 0$ (or equivalently, $N \rightarrow \infty$ ), $\widehat{G}$ approaches the correlation function (2.14) in the $M=0$ BTZ geometry, denoted in the graph by dashed lines.

In Lorentzian signature we set

$$
w=\phi-t, \quad \bar{w}=\phi+t .
$$

The correlator $G(w, \bar{w})=G(t, \phi)$ then diverges at $w=k \pi / 2$ or $\bar{w}=k \pi / 2$ with $k \in \mathbb{Z}$. This divergence is a physical one, since on a finite cylinder a particle periodically returns to the same spatial location. Therefore, in order to make the temporal behaviour of the correlation function more transparent, it is useful to remove this divergence. So, let us define the regularized correlator $\widehat{G}(t, \phi)$ by dividing $G(t, \phi)$ by the vacuum correlation function of the probe graviton operator:

$$
\begin{aligned}
\widehat{G}(t, \phi) & \equiv-16 \sin ^{2} \frac{w}{2} \sin ^{2} \frac{\bar{w}}{2} G(t, \phi) \\
& =\frac{1}{N} \sum_{n=1}^{\infty} \frac{n N_{n}}{\left(n \sin \frac{t}{n}\right)^{2}}\left[\sin ^{2} \frac{w}{2}+\sin ^{2} \frac{\bar{w}}{2}-\frac{2 \sin t \sin \frac{w}{2} \sin \frac{\bar{w}}{2}}{n \tan \frac{t}{n}}\right] .
\end{aligned}
$$

Plugging in the representative distribution of constituent twists for microstates with $J=0$ (3.5) into the regularized correlator (4.13) we obtain figure 1. As one can see from this graph, the correlator decays rapidly at initial times $(t \lesssim \pi)$, and at later times exhibits a quasi-periodic behaviour. Quasi-periodicity is not surprising; it is expected on general grounds in a system with a finite number of degrees of freedom. Furthermore, one sees that, in the $\beta \rightarrow 0$ (or equivalently $N \rightarrow \infty$ ) limit, $\widehat{G}$ approaches a certain limit shape. As we will discuss below, 
the limit shape in the $N \rightarrow \infty$ limit turns out to be the correlation function (2.14) in the $M=0 \mathrm{BTZ}$ geometry.

\subsection{Effective geometry of microstates with $J=0$}

Now consider the correlation function (4.9) of a general bosonic non-twist operator:

$$
\left\langle\mathcal{A}\left(w_{1}\right) \mathcal{A}\left(w_{2}\right)\right\rangle_{\Sigma}=\frac{1}{N} \sum_{n} n N_{n} \sum_{k=0}^{n-1} \frac{C}{\left[2 n \sin \left(\frac{w-2 \pi k}{2 n}\right)\right]^{2 h}\left[2 n \sin \left(\frac{\bar{w}-2 \pi k}{2 n}\right)\right]^{2 \tilde{h}}} .
$$

Let us study the relative size of the contributions to this from terms with different $n$. The contributions come multiplied by $N_{n}$, which is $\frac{8 n}{\sinh \beta n}$ for the typical microstates with $J=0$ (equation (3.5)). Because of the suppression by the $\sinh \beta n$, the values of $n$ that make substantial contributions to the correlation function (4.9) are $n \lesssim 1 / \beta \sim \sqrt{N}$. Thus there are $O(\sqrt{N})$ twists that make a significant contribution. Now observe that for any $\gamma<1 / 2$, the number of twists with $n \lesssim N^{\gamma}$ is parametrically smaller than $\sqrt{N}$. Indeed, the ratio vanishes as $N \rightarrow \infty$. In this sense we can say that in the $N \rightarrow \infty$ limit, (4.14) is dominated by twists scaling as $n \sim \sqrt{N}$.

Next, for any $n \geqslant 1$, when $t \ll n$ we can approximate the sum on $k$ as

$$
\begin{aligned}
& \sum_{k=0}^{n-1} \frac{1}{\left[2 n \sin \left(\frac{w-2 \pi k}{2 n}\right)\right]^{2 h}\left[2 n \sin \left(\frac{\bar{w}-2 \pi k}{2 n}\right)\right]^{2 \tilde{h}}} \\
& \quad \approx \sum_{k=-\infty}^{\infty} \frac{1}{(w-2 \pi k)^{2 h}(\bar{w}-2 \pi k)^{2 \tilde{h}}} \quad(t \ll n),
\end{aligned}
$$

where we assumed $h+\tilde{h}=$ even.

Putting the above statements together, we arrive at the following conclusion: for sufficiently early times

$$
t \ll t_{c}=\mathcal{O}(\sqrt{N}),
$$

the correlation function (4.14) can be approximated by

$$
\begin{aligned}
\left\langle\mathcal{A}\left(w_{1}\right) \mathcal{A}\left(w_{2}\right)\right\rangle_{\Sigma} & \approx \frac{1}{N} \sum_{n} n N_{n} \sum_{k=-\infty}^{\infty} \frac{C}{(w-2 \pi k)^{2 h}(\bar{w}-2 \pi k)^{2 \tilde{h}}} \\
& =\sum_{k=-\infty}^{\infty} \frac{C}{(w-2 \pi k)^{2 h}(\bar{w}-2 \pi k)^{2 \tilde{h}}} .
\end{aligned}
$$

This is precisely the bulk correlation function in the naive geometry, or the $M=0$ BTZ black hole (compare with (2.14)) for $h=\tilde{h}=1$ ). Therefore, in the orbifold CFT approximation, the emergent effective geometry of the D1-D5 system is the $M=0 \mathrm{BTZ}$ black hole geometry. The description in terms of this effective geometry is valid until $t \sim t_{c}$, which goes to infinity as $N \rightarrow \infty$. In the special case $h=\tilde{h}=1$, the summation (4.16) yields (2.14). In this case, we indeed saw in figure 1 that the $\beta \rightarrow 0$ limit of the correlation function is given by (2.14) (or (4.16)).

Note that in (4.16) the sum over the twists $n$ factors out. Thus, for $t<t_{c}$ we show that the correlation function is largely independent of the detailed microscopic distribution of twists. It is this universal response that reproduces the physics of the $M=0 \mathrm{BTZ}$ black hole. After $t \sim t_{c}$, the approximation (4.16) breaks down, and the correlation function starts to show random-looking, quasi-periodic behaviour (see figure 1). The form of the correlation function 
in this regime will depend on the precise form of the individual microstate, no matter how close it is to the representative state (3.5).

The $\beta \rightarrow 0$ limit corresponding to the $M=0$ BTZ black hole yields a correlator which decays to zero at large times as $1 / t^{2}$. By contrast, the microstate correlators exhibit quasiperiodic fluctuations around a nonzero mean value. Numerical analysis indicates that this mean value scales as $\frac{1}{\sqrt{N}}$ for $h=\tilde{h}=1$. For an ordinary finite size, finite temperature system, one expects the mean value to be of order $\mathrm{e}^{-c S}$ where $S$ is the entropy and $c$ is of order 1 . This behaviour arises because typical interactions can explore the entire phase space of the system. The fact that we observe power law rather than exponential behaviour is likely to be a result of working in the free orbifold limit of the CFT and probing the system with only non-twist operators. Under these conditions, the full space of states does not come into play in determining a correlation function. For example, the non-twist operators cannot see the full structure of the microstate, for instance the relative phases between different twist components, and so might be expected to exhibit larger correlations at late times. For this reason, it would be very instructive to repeat our analysis for twist operators, although this is technically much more challenging.

A finite $N$ microstate correlator will exhibit exact periodicity in time because only a finite number of frequencies appear in the Fourier expansion. The frequencies are $\omega_{n}=\frac{n}{N}, n=1,2, \ldots, N$. Let $L(N)$ denote the least common multiple of $(1,2, \ldots, N)$. The correlator is then periodic with period $\Delta t=2 \pi N L(N)$. The large $N$ behaviour of $L(N)$ is $L(N) \sim \mathrm{e}^{N}$, and therefore

$$
\Delta t \sim N \mathrm{e}^{N} .
$$

Our correlators have been computed in the canonical ensemble in which the summation over $n$ extends past $N$ up to infinity, and so we will not see this exact periodicity. On the other hand, due to the exponential suppression of the distribution function $N_{n}$ the deviation from exact periodicity is tiny for large $N$. As was argued above, and as can be confirmed numerically, one finds that for large $N$ the large time behaviour of the correlator is unaffected if we truncate the sum over $n$ at $n_{\max }=c \sqrt{N}$, for $c$ of order unity. Taking this into account, we see that our correlators will exhibit approximate periodicity with period

$$
\Delta t \sim \mathrm{e}^{c \sqrt{N}}=\mathrm{e}^{\tilde{c} S},
$$

where $S=2 \pi \sqrt{2} \sqrt{N}$ is the entropy. This timescale is the so-called Poincare recurrence time, over which generic finite size thermal systems are expected to exhibit approximate periodicity.

Fermionic probes. In the above, we restricted ourselves to bosonic probes, but we obtain the same effective geometry even if we probe the microstate with operators that contain fermions. For example, let $\mathcal{A}=\Psi^{s^{\prime}} \widetilde{\Psi}^{\widetilde{s}^{\prime}}$ as defined in (A.4). From (A.40), we obtain

$$
\left\langle\mathcal{A}_{A} \mathcal{A}_{B}\right\rangle_{\sigma_{n}^{\widetilde{s}}}=\left\langle\left.\left[\Psi_{A}^{s^{\prime}} \widetilde{\Psi}_{A}^{\widetilde{s}^{\prime}}\left(w_{1}\right)\right]^{\dagger} \Psi_{B}^{s^{\prime}} \widetilde{\Psi}_{B}^{\widetilde{s}^{\prime}}\left(w_{2}\right)\right|_{\sigma_{\sigma^{\widetilde{s}}}}=\frac{\mathrm{e}^{\mathrm{i} s s^{\prime} w / 2 n-i \widetilde{\mathrm{is}} \bar{s}^{\prime} \bar{w} / 2 n}}{\left(2 n \sin \frac{w}{2 n}\right)\left(2 n \sin \frac{\bar{w}}{2 n}\right)} .\right.
$$

If we sum over copies,

$$
\begin{aligned}
\sum_{A=1}^{n}\left\langle\mathcal{A}_{A} \mathcal{A}_{1}\right\rangle_{\sigma_{n}^{\widetilde{s}}} & =\sum_{k=0}^{n-1} \frac{\mathrm{e}^{\mathrm{i} s s^{\prime}(w+2 \pi k) / 2 n-\mathrm{i} \widetilde{S^{\prime}}(\bar{w}+2 \pi k) / 2 n}}{\left(2 n \sin \frac{w+2 \pi k}{2 n}\right)\left(2 n \sin \frac{\bar{w}+2 \pi k}{2 n}\right)} \\
& \approx \sum_{k=-\infty}^{\infty} \frac{1}{(w+2 \pi k)(\bar{w}+2 \pi k)} \quad(t \ll n) .
\end{aligned}
$$


Other twist operators $\sigma_{n}^{\widetilde{\alpha} \widetilde{\beta}}, \tau_{n}^{\widetilde{\alpha}}, \tau_{n}^{\widetilde{\alpha s}}$ give different correlation functions, but they are all identical to (4.20) for $t \ll n$,

$$
\sum_{A=1}^{n}\left\langle\mathcal{A}_{A} \mathcal{A}_{1}\right\rangle_{\sigma_{n}^{\widehat{\mu}}} \approx \sum_{k=-\infty}^{\infty} \frac{1}{(w+2 \pi k)(\bar{w}+2 \pi k)} \quad(t \ll n),
$$

where $\sigma_{n}^{\widehat{\mu}}$ can be any of the twist operators $\sigma_{n}^{s \widetilde{s}}, \sigma_{n}^{\widetilde{\alpha} \widetilde{\beta}}, \tau_{n}^{s \widetilde{\alpha}}, \tau_{n}^{\widetilde{\alpha s}}$. Plugging this result into (4.5), we conclude that

$$
\begin{aligned}
\left\langle\mathcal{A}\left(w_{1}\right) \mathcal{A}\left(w_{2}\right)\right\rangle_{\Sigma} & =\left\langle\left[\Psi_{A}^{s^{\prime}} \widetilde{\Psi}_{A}^{\widetilde{s}^{\prime}}\left(w_{1}\right)\right]^{\dagger} \Psi_{B}^{s^{\prime}} \widetilde{\Psi}_{B}^{\widetilde{s}^{\prime}}\left(w_{2}\right)\right\rangle_{\Sigma} \\
& \approx \sum_{k=-\infty}^{\infty} \frac{1}{(w-2 \pi k)(\bar{w}-2 \pi k)} \quad\left(t \ll t_{c}\right) .
\end{aligned}
$$

This is again the correlation function in the $M=0$ BTZ black hole geometry. Therefore, we conclude that the effective geometry of the D1-D5 system in the orbifold CFT approximation is the $M=0 \mathrm{BTZ}$ black hole geometry for any non-twist probe operators, bosonic or fermionic. The description by this effective geometry breaks down at $t=t_{c}=\mathcal{O}(\sqrt{N})$.

Gravitational origin of the effective geometry. We can also argue that the effective geometry for the ensemble with $J=0$ should be the $M=0$ BTZ black hole by using the Lunin-Mathur metric (2.8). Assume that the profile $F_{i}(v)$ is a random superposition of small-amplitude, high-frequency oscillations that is much smaller than the asymptotic AdS radius:

$$
|\mathbf{F}(v)| \ll \ell \sim N^{1 / 4} .
$$

Then, for $r \sim \ell \gg|\mathbf{F}(v)|$,

$$
\begin{aligned}
f_{5} & =\frac{Q_{5}}{L} \int_{0}^{L} \mathrm{~d} v \frac{1}{|\mathbf{x}-\mathbf{F}(v)|^{2}} \approx \frac{Q_{5}}{r^{2}} \\
f_{1} & =\frac{Q_{5}}{L} \int_{0}^{L} \mathrm{~d} v \frac{|\dot{\mathbf{F}}(v)|^{2}}{|\mathbf{x}-\mathbf{F}(v)|^{2}} \approx \frac{1}{r^{2}} \frac{Q_{5}}{L} \int_{0}^{L} \mathrm{~d} v|\dot{\mathbf{F}}(v)|^{2}=\frac{Q_{1}}{r^{2}} \\
A_{i} & =\frac{Q_{5}}{L} \int_{0}^{L} \mathrm{~d} v \frac{\dot{F}_{i}(v)}{|\mathbf{x}-\mathbf{F}(v)|^{2}} \approx 0
\end{aligned}
$$

where in the second line we used (2.9), and in the third line $A_{i}(x)$ vanishes because $F_{i}(v)$ is random. So the metric (2.8) is

$$
\mathrm{d} s^{2}=-\frac{r^{2}}{\ell^{2}} \mathrm{~d} t^{2}+\frac{r^{2}}{\ell^{2}} \mathrm{~d} y^{2}+\frac{\ell^{2}}{r^{2}}\left(\mathrm{~d} r^{2}+r^{2} \mathrm{~d} \Omega_{3}^{2}\right)+\sqrt{\frac{Q_{1}}{Q_{5}}} \mathrm{~d} s_{T^{4}}^{2}
$$

with $\ell=\left(Q_{1} Q_{5}\right)^{1 / 4}$. This is indeed the direct product of the $M=0$ BTZ black hole (2.13), and $S^{3} \times T^{4}$, if one sets $y \rightarrow R \phi, r \rightarrow \ell r / R$. One can check that the condition (4.23) is satisfied from the microscopic theory, as follows. If the typical frequency and amplitude are $\omega$ and $a$, respectively, then $|\mathbf{F}| \sim a,|\dot{\mathbf{F}}| \sim a \omega$. We can relate $\omega, a$ with the microscopic quantities $n, N_{n}$ as $\omega \sim n, a \sim N_{n}^{1 / 2}$. Recall that the typical twist is $n \sim N^{1 / 2} \sim 1 / \beta$. For $n \sim 1 / \beta, N_{n}$ is $N_{n}=\frac{8}{\sinh (\beta n)}=\mathcal{O}(1)$ from (3.5). Therefore, $\omega \sim N^{1 / 2}, a \sim N^{0}$. This indeed satisfies (4.23).

\subsection{Effective geometry of microstates with $J \neq 0$}

As we saw in section 3.3, the ensemble with $J \neq 0$ becomes in the large $N$ limit a 'direct product' of the Bose-Einstein condensate $\left(\sigma_{1}^{s \widetilde{s}}\right)^{|J|}$ with $s=\widetilde{s}=\mp$, and an ensemble with 
level $\tilde{N}=N-|J|$ and no angular momentum. Therefore, from the general formula (4.5), one sees that the correlation function for this ensemble is a sum of the correlation function in the Bose-Einstein condensate background and the one for the ensemble with level $\widetilde{N}=N-|J|$ and no angular momentum.

Specifically, consider a bosonic non-twist operator $\mathcal{A}$. Plugging the typical distribution (3.9) into the formula (4.9),

$$
\begin{aligned}
& \left\langle\mathcal{A}\left(w_{1}\right) \mathcal{A}\left(w_{2}\right)\right\rangle_{\Sigma} \\
& =\frac{|J|}{N} \frac{C}{\left(2 \sin \frac{w}{2}\right)^{2 h}\left(2 \sin \frac{\bar{w}}{2}\right)^{2 \tilde{h}}}+\frac{1}{N} \sum_{n} n \tilde{N}_{n} \sum_{k=0}^{n-1} \frac{C}{\left[2 n \sin \left(\frac{w-2 \pi k}{2 n}\right)\right]^{2 h}\left[2 n \sin \left(\frac{\bar{w}-2 \pi k}{2 n}\right)\right]^{2 \tilde{h}}} \\
& \approx \frac{|J|}{N} \frac{C}{\left(2 \sin \frac{w}{2}\right)^{2 h}\left(2 \sin \frac{\bar{w}}{2}\right)^{2 \tilde{h}}}+\left(1-\frac{|J|}{N}\right) \sum_{k=-\infty}^{\infty} \frac{C}{(w-2 \pi k)^{2 h}(\bar{w}-2 \pi k)^{2 \tilde{h}}} \quad\left(t \ll t_{c}\right) \\
& =\frac{|J|}{N}\langle\mathcal{A} \mathcal{A}\rangle_{\mathrm{BEC}}+\left(1-\frac{|J|}{N}\right)\langle\mathcal{A} \mathcal{A}\rangle_{M=0 \mathrm{BTZ}}
\end{aligned}
$$

The critical time $t_{c}$ is now given by

$$
t_{c}=\mathcal{O}(\sqrt{N-|J|}) \text {. }
$$

The first term in (4.28), which arises from the Bose-Einstein condensate (BEC), is proportional to the correlation function of $\mathcal{A}$ computed in global $\mathrm{AdS}_{3}$. This happens because the condensate is $\left(\sigma_{1}^{s \widetilde{s}}\right)^{|J|}, s=\widetilde{s}=\mp$, and the three-dimensional part of the microstate geometry associated with this operator by itself is simply global $\operatorname{AdS}_{3}$ with a scale $\ell \sim|J|^{1 / 4}$, as described by [36-38]. Actually, the total 10-dimensional geometry is more complicated because of the nontrivial Wilson line coming from the internal $S^{3}$, but the bosonic operator $\mathcal{A}$ does not sense this extra structure. On the other hand, fermionic $\mathcal{A}$ does see this structure, as we will see below.

Hence the 'effective geometry' for $t<t_{c}$ appears to be a weighted average of global $\mathrm{AdS}_{3}$ (with a nontrivial Wilson line) and the $M=0 \mathrm{BTZ}$. The linear summation in (4.28) appears because in the orbifold CFT the simple class of non-twist probes has correlation functions that are simply linear summations of the responses in the individual constituent twist states (4.28). Of course as $|J| \rightarrow N$, the typical microstate operator found in (3.8) becomes precisely the operator corresponding to global $\mathrm{AdS}_{3}$ (with a Wilson line) in [36-38]. Thus the response (4.28) is simply a weighted sum of the expected responses in the $J=0$ and $|J|=N$ limits.

Correlation functions involving fermionic operators can be evaluated similarly. For example, let us take $\mathcal{A}=\Psi^{s^{\prime}} \widetilde{\Psi}^{\widetilde{s}^{\prime}}$ as before. From (4.9), (4.19) and (4.21), we obtain

$$
\begin{aligned}
\left\langle\mathcal{A}_{A} \mathcal{A}_{B}\right\rangle_{\Sigma} & \approx \frac{|J|}{N} \frac{\mathrm{e}^{\mathrm{i} s\left(s^{\prime} w-\widetilde{s}^{\prime} \bar{w}\right) / 2}}{\left(2 \sin \frac{w}{2}\right)\left(2 \sin \frac{\bar{w}}{2}\right)}+\left(1-\frac{|J|}{N}\right) \sum_{k=-\infty}^{\infty} \frac{1}{(w-2 \pi k)(\bar{w}-2 \pi k)} \quad\left(t \ll t_{c}\right) \\
& =\frac{|J|}{N}\langle\mathcal{A} \mathcal{A}\rangle_{\mathrm{BEC}}+\left(1-\frac{|J|}{N}\right)\langle\mathcal{A} \mathcal{A}\rangle_{M=0 \mathrm{BTZ}}
\end{aligned}
$$

where $s=\operatorname{sign}(J)$. Again the 'effective geometry' appears to be a weighted average.

The Bose-Einstein condensate part $\langle\mathcal{A} \mathcal{A}\rangle_{\mathrm{BEC}}$ of the bosonic correlator (4.28) did not care whether the condensate is made of $\sigma_{1}^{s \widetilde{s}}$ with $s=\widetilde{s}=-1$ or $s=\widetilde{s}=+1$, whereas the fermionic one (4.30) does depend on what the condensate is made of through its dependence on $s=\operatorname{sign}(J)$. This reflects the fact that the three-dimensional geometry corresponding to $\left(\sigma_{1}^{s \widetilde{s}}\right)^{N}$ with $s=\widetilde{s}=-1$ and the one with $s=\widetilde{s}=+1$ are both global AdS 3 but differ in the nontrivial Wilson line in the internal $S^{3}$ [36-38]. Bosonic probes are not charged under the 
relevant $U(1)$, and thus its correlator is independent of what the condensate is made of. On the other hand, fermionic probes are charged under the $U(1)$, and its correlator depends on what the condensate is made of.

Do the above results mean that the emergent geometry is a superposition of two classical geometries? Below we will use the Lunin-Mathur solution (2.7) to argue that this should not be the case and that the emergent geometry should be a singular zero-horizon limit of the black ring [51].

The effective geometry should be a black ring. Assuming $J>0$ and $J=\mathcal{O}(N)$, the typical state of the ensemble with $J \neq 0$ is given by (3.8). In the language of the FP system, $\left(\alpha_{-1}^{+}\right)^{J}$ corresponds to an F1 worldvolume that makes a circle with radius $\sim \sqrt{J}=\mathcal{O}\left(N^{1 / 2}\right)$ in the 1-2 plane. The remaining part $\prod_{n=1}^{\infty}\left[\prod_{i}\left(\alpha_{-n}^{i}\right)^{N_{n i}}\left(\psi_{-n}^{i}\right)^{N_{n i}^{\prime}}\right]$ adds fluctuations around this circular profile. By an argument similar to that given at the end of the last subsection, the typical frequency and amplitude of the fluctuations are estimated to be $n \sim \sqrt{N-J}=\mathcal{O}\left(N^{1 / 2}\right)$ and $N_{n}^{1 / 2}=\mathcal{O}\left(N^{0}\right)$, respectively.

This motivates the following profile function $\mathbf{F}(v)$ of the D1-D5 metric (2.7). Namely, we assume that the profile $\mathbf{F}(v)$ is a circle $\mathbf{F}^{(0)}$ with random, small-amplitude, high-frequency fluctuations $\delta \mathbf{F}$ around it:

$\mathbf{F}=\mathbf{F}^{(0)}+\delta \mathbf{F}, \quad\left\{\begin{array}{l}F_{1}^{(0)}+\mathrm{i} F_{2}^{(0)}=a \mathrm{e}^{\mathrm{i} \omega v}, \\ F_{3}^{(0)}=F_{4}^{(0)}=0,\end{array} \quad \omega=\frac{2 \pi}{L}=\frac{R}{Q_{5}}\right.$.

From the above analysis, the amplitude of the fluctuation $\delta \mathbf{F}$ is much smaller than the size of the circle or the AdS radius:

$|\delta \mathbf{F}|=\mathcal{O}\left(N^{0}\right) \ll\left|\mathbf{F}^{(0)}\right|=a=\mathcal{O}\left(N^{1 / 2}\right), \quad|\delta \mathbf{F}|=\mathcal{O}\left(N^{0}\right) \ll \ell=\mathcal{O}\left(N^{1 / 4}\right)$.

On the other hand, the derivatives of $\mathbf{F}^{(0)}$ and $\delta \mathbf{F}$ are of the same order of magnitude:

$$
|\delta \dot{\mathbf{F}}| \sim n N_{n}^{1 / 2}=\mathcal{O}\left(N^{1 / 2}\right), \quad\left|\dot{\mathbf{F}}^{(0)}\right|=a \omega=\mathcal{O}\left(N^{1 / 2}\right) .
$$

Using these relations, the harmonic functions in (2.7) are approximated for large $N$ as follows ${ }^{6}$,

$f_{5} \approx \frac{Q_{5}}{L} \int_{0}^{L} \mathrm{~d} v \frac{1}{\left|\mathbf{x}-\mathbf{F}^{(0)}\right|^{2}}=\frac{Q_{5}}{\Sigma}$,

$f_{1} \approx \frac{Q_{5}}{L}\left(a^{2} \omega^{2}+|\delta \dot{\mathbf{F}}|^{2}\right) \int_{0}^{L} \mathrm{~d} v \frac{1}{\left|\mathbf{x}-\mathbf{F}^{(0)}\right|^{2}}=\frac{Q_{1}}{\Sigma}$,

$A_{1}+\mathrm{i} A_{2} \approx \frac{Q_{5}}{L} \int_{0}^{L} \mathrm{~d} v \frac{\mathrm{i} a \omega \mathrm{e}^{\mathrm{i} \omega v}}{\left|\mathbf{x}-\mathbf{F}^{(0)}\right|^{2}}, \quad$ therefore $\quad A_{\psi}=\frac{2 a^{2} Q_{5} \omega s^{2}}{\Sigma\left(\Sigma+s^{2}+w^{2}+a^{2}\right)}$,

where

$x_{1}+\mathrm{i} x_{2}=s \mathrm{e}^{\mathrm{i} \psi}, \quad x_{3}+\mathrm{i} x_{4}=w \mathrm{e}^{\mathrm{i} \phi}, \quad \Sigma=\sqrt{\left[(s+a)^{2}+w^{2}\right]\left[(s-a)^{2}+w^{2}\right]}$.

In the second line of (4.34), the cross term $\mathbf{F}^{(0)} \cdot \delta \mathbf{F}$ was dropped because $\delta \mathbf{F}$ is fluctuating randomly. Also in the second line, because $|\delta \dot{\mathbf{F}}|^{2}$ is fluctuating with length scale much smaller than $a$, we can replace it with its average and take it out of the integral (so, $|\delta \dot{\mathbf{F}}|^{2}$ there really means the average). We also used relation (2.9):

$$
Q_{1}=\frac{Q_{5}}{L} \int_{0}^{L} \mathrm{~d} v|\dot{\mathbf{F}}|^{2} \approx\left(a^{2} \omega^{2}+|\delta \dot{\mathbf{F}}|^{2}\right) Q_{5}
$$

In the third line of (4.34), the term containing $\delta \dot{\mathbf{F}}$ was dropped because it is fluctuating randomly. It is convenient to go to the $(x, y, \psi, \phi)$ coordinate system [51] with $R=a$,

6 This metric was studied in [52] using a different ansatz of the profile function $\mathbf{F}(v)$. Recent analysis of this metric from the bubbling AdS viewpoint of [33] can be found in [53]. 
defined by

$$
s=\frac{\sqrt{y^{2}-1}}{x-y} R, \quad w=\frac{\sqrt{1-x^{2}}}{x-y} R .
$$

In this coordinate system, $A_{i}, B_{i}, \Sigma$ can be written as

$$
A_{\psi}=\frac{Q_{5} \omega}{2}(-1-y), \quad B_{\phi}=\frac{Q_{5} \omega}{2}(1+x), \quad \Sigma=\frac{2 R^{2}}{x-y} .
$$

Plugging these into (2.7), one obtains the metric

$\mathrm{d} s^{2}=\frac{\Sigma}{\ell^{2}}\left[-\left(\mathrm{d} t+\frac{Q_{5} \omega}{2}(-1-y) \mathrm{d} \psi\right)^{2}+\left(\mathrm{d} y+\frac{Q_{5} \omega}{2}(1+x) \mathrm{d} \phi\right)^{2}\right]+\frac{\ell^{2}}{\Sigma} \mathrm{d} s_{4}^{2}+\sqrt{\frac{Q_{1}}{Q_{5}}} \mathrm{~d} s_{T^{4}}^{2}$,

where $\ell \equiv\left(Q_{1} Q_{5}\right)^{1 / 4}$. This is the metric of the supersymmetric black ring [51, 54] with charges $\left(Q_{1}, Q_{2}, Q_{3}\right)=\left(Q_{1}, Q_{5}, 0\right)$, dipole charges $\left(q_{1}, q_{2}, q_{3}\right)=\left(0,0, Q_{5} \omega\right)$ and radius $R=a$. For these charges, the horizon area and thus the Bekenstein-Hawking entropy vanish. The angular momentum of this singular black ring satisfies

$$
J_{\psi}=R^{2} q_{3} \leqslant \frac{Q_{1} Q_{2}}{q_{3}} \equiv J_{\psi, \max } .
$$

If this inequality is saturated, the singular black ring becomes the regular D1-D5 $\rightarrow \mathrm{kk}$ geometry. However, in the present case,

$$
J_{\psi}=a^{2} Q_{5} \omega, \quad J_{\psi, \max }=\frac{Q_{1} Q_{5}}{Q_{5} \omega}=a^{2} Q_{5} \omega\left(1+\frac{|\delta \dot{\mathbf{F}}|^{2}}{a^{2} \omega^{2}}\right) .
$$

So, the equality in (4.40) does not hold and the geometry (4.39) describes a singular, zerohorizon limit of the black ring.

The above argument suggests that the effective geometry for the ensemble with $J \neq 0$ is the singular, zero-horizon limit of the black ring $(4.39)^{7}$. The description by this effective geometry should be valid up to the critical time $t_{c}$ (4.29), which goes to infinity as $N \rightarrow \infty$. In order to prove the above statement, one should compute the bulk-boundary propagator in the singular black ring geometry (4.39) and show that it leads to the boundary CFT correlation function (4.28), (4.30).

\section{Discussion}

The puzzles regarding the black hole information paradox are all traceable to the fact that we do not have an adequate understanding of the relation between geometry and entropy. In the boundary CFT description of black holes, we can choose to work either with individual microstates or with an ensemble, and we understand that entropy arises from the coarsegraining associated with defining the ensemble. In practice, the ensemble usually yields results to the accuracy we desire, and the existence of the underlying microstate description tells us that there is no possibility of information loss at a fundamental level.

We lack a similar understanding in the bulk. If the black hole is to be thought of as an ensemble, we need to specify precisely the elements of the ensemble. One logical possibility is that the bulk description is intrinsically coarse-grained, and that microstates can

7 This is reminiscent of the proposal by [55] that the CFT microstate of the black ring with non-vanishing horizon is made of two parts, where the first part is made of small effective strings of identical length, while the second part is made of a single long string and responsible for the whole entropy. 
only be found in the boundary CFT. An alternative picture, advocated by Mathur, is that bulk microstates are to be described as new horizon-free geometries differing from the black hole at the horizon scale. Some evidence for the latter has accumulated, but the question remains open.

Here, we have studied some of these issues in the simple context of the D1-D5 CFT at the free orbifold point. On the one hand, a large class of microstate geometries are known, and on the other hand there is an effective 'black hole' geometry describing their 'average'. We essentially tried to make this last sentence precise by comparing CFT correlation functions computed in typical microstates to bulk correlation functions computed in the 'black hole' geometry. The agreement we found, as well as its breakdown at late times, provides evidence for the picture of black holes as the effective description of more fundamental underlying structures. Although the 'black hole' in this case has vanishing horizon size, it does display some of the hallmarks of real black holes, such as the decay of late time correlators.

If black holes in general represent effective coarse-grained descriptions of underlying microstate geometries, it naturally explains why one cannot see quasi-periodicity and Poincaré recurrence by summing over the $S L(2, \mathbb{Z})$ family of BTZ black holes as was pursued in $[9,11]$. This is analogous to the fact that, after replacing a gas of molecules by its effective coarse-grained description, i.e., a dissipative continuum, one does not expect to be able to see quasi-periodicity or Poincaré recurrence in the correlation function describing a particle scattered in the gas.

It would be interesting to try to repeat our calculations in the context of the D1-D5 system on $\mathrm{K} 3$ rather than $T^{4}$. In the $\mathrm{K} 3$ case, it has been found that higher derivative terms in the supergravity Lagrangian lead to a nonzero size horizon whose Bekenstein-Hawking-Wald entropy agrees with that of the CFT $[15,16]$. Furthermore, one can still write down a large class of microscopic geometries which contribute to the entropy [56]. The complication is that the sigma model is no longer free, and so the computation of CFT correlators is not as straightforward. But the goal would be to show how the nonzero horizon size manifests itself in CFT correlators. Alternatively, perhaps a horizon could be found even in the $T^{4}$ case once interactions are included.

Another useful endeavour would be to compare bulk correlators computed in the known microstate geometries of the D1-D5 system to the microscopic CFT correlators we have computed here. This can easily be done for the simplest class of states, namely those corresponding to the twist operator $\sigma=\left[\sigma_{n}^{s \widetilde{s}}\right]^{N / n}, s=\widetilde{s}=-1$. In this case, the bulk geometries are simply the conical defects (2.10), and we saw that this gives precise agreement between bulk and boundary correlators. But for more general states the bulk geometry is no longer just an orbifold, and the bulk correlators will be much more complicated. On the other hand, the CFT correlators continue to be expressed as a sum of simple contributions. This suggests that either the bulk geometries can also somehow be thought of as being built up out of simple geometries, or alternatively that working at the free orbifold point of the CFT is simply inadequate.

In this paper, we studied correlation functions of non-twist operators, but it would be very interesting to consider twist operators. This would allow much greater sensitivity to the microstate structure. Non-twist operators see the states as built out of decoupled components corresponding to the given cycles, and this led to the correlators taking the form of a sum over relatively simple contributions from each component. This will no longer be the case when twist operators are used to probe the state, and the results are expected to be much more complicated. This extra information could potentially be used to map out the bulk geometry in much greater detail. 


\section{Acknowledgments}

We would like to thank Jan de Boer, Hiroshi Fujisaki, Norihiro Iizuka, Vishnu Jejjala, Oleg Lunin, Joan Simon, Sanefumi Moriyama, and Hirosi Ooguri for valuable discussions. We would also like to thank the organizers of the workshop on Quantum Theory of Black Holes at the Ohio State University, where this work was initiated, the Workshop on Gravitational Aspects of String Theory at the Fields Institute, and Strings 2005, for stimulating environments. MS would like to thank Norihiro Iizuka for collaboration in [21] and helpful discussions, and the Theoretical High Energy Physics group at the University of Pennsylvania for hospitality. PK was supported in part by NSF grant no PHY-0099590. MS was supported in part by Department of Energy grant no DE-FG03-92ER40701 and a Sherman Fairchild Foundation postdoctoral fellowship. VB was supported in part by the DOE under grant no DE-FG0295ER40893, by the NSF under grant no PHY-0331728 and by an NSF Focused Research Grant DMS0139799.

\section{Appendix A. D1-D5 CFT}

In this appendix, we present a complete review of the relevant aspects of the D1-D5 CFT, in particular chiral primary fields and the corresponding $\mathrm{R}$ (amond) ground states related by spectral flow. We will compute correlation functions of non-twist operators in the $\mathrm{R}$ ground states, which are related via AdS/CFT to supergravity amplitudes in $\mathrm{AdS}_{3} \times S^{3}$. References on $S_{N}$ orbifold CFTs and methods for computing correlation functions in them include [57-67]. Below we will closely follow the argument of [66, 68, 69] and the notation of [66]. For a more detailed explanation of the covering space method and the NS sector chiral primaries, see $[64,66]$.

The main results from this appendix that are used in the main text of this paper are the bosonic two-point function (A.33) and the fermionic two-point functions (A.39)-(A.41). The two-point function for the general state (A.23) can be computed using (A.28) and (A.29). We will derive these using orbifold CFT machinery, but the final results for the two-point function are simple and intuitive, and can be obtained more simply by just taking into account the fact that the effective length of the CFT cylinder undergoes a rescaling. However, the detailed machinery described below is necessary for further computation of more general correlation functions, particularly those involving twist operators as probes.

\section{A.1. D1-D5 system}

Consider type IIB string theory on $\mathbb{R}_{t} \times \mathbb{R}^{4} \times S^{1} \times T^{4}$ with $N_{1}$ D1-branes and $N_{5}$ D5-branes. The D1-branes are wound on $S^{1}$ and smeared over $T^{4}$, and the D5-branes are wrapped on $S^{1} \times T^{4}$. We denote by $x^{0}$ the time direction $\mathbb{R}_{t}$; by $x^{i}(i=1,2,3,4)$ the $\mathbb{R}^{4}$ directions; by $x^{5}$ the $S^{1}$ direction; and by $x^{a}(a=6,7,8,9)$ the $T^{4}$ directions (see table 1$)$. The low energy worldvolume dynamics of the D1-D5 system is described by a $(1+1)$-dimensional $\mathcal{N}=(4,4)$ SCFT in the RR sector, where the two dimensions come from the $x^{0,5}$ directions [39-42]. This theory has $S O(4)_{E} \cong S U(2)_{R} \times \widehat{S U(2)_{R}} R$-symmetry, which originates from the rotational symmetry in the transverse directions $x^{i}, i=1,2,3,4$. On the other hand, the rotation in the longitudinal directions $x^{a}, a=6,7,8,9$ leads to $S O(4)_{I} \cong S U(2)_{I} \times \widetilde{S U(2)_{I}}$ symmetry. Actually, $T^{4}$ breaks the latter symmetry, but it can still be used for classifying the states in the theory. 
Table 1. Configuration of D-branes.

\begin{tabular}{ccccccccccc}
\hline & 0 & 1 & 2 & 3 & 4 & 5 & 6 & 7 & 8 & 9 \\
\hline D1 & $\bigcirc$ & $\cdot$ & $\cdot$ & $\cdot$ & $\cdot$ & $\bigcirc$ & $\sim$ & $\sim$ & $\sim$ & $\sim$ \\
D5 & $\bigcirc$ & $\cdot$ & $\cdot$ & $\cdot$ & $\cdot$ & $\bigcirc$ & $\bigcirc$ & $\bigcirc$ & $\bigcirc$ & $\bigcirc$ \\
\hline
\end{tabular}

The CFT is a sigma model whose target space is the symmetric product $\mathcal{M}_{0}=\left(T^{4}\right)^{N} / S_{N}$, where $S_{N}$ is the permutation group of order $N$. We put

$$
N=N_{1} N_{5}
$$

More precisely, the target space is not the symmetric product $\mathcal{M}_{0}$ but a deformation of it; the sigma model has marginal deformations, which one has to turn on in order for the CFT to precisely correspond to the supergravity side. $\mathcal{M}_{0}$ is a special point in the moduli space of the CFT called the orbifold point, where the CFT becomes free. This situation is very similar to the situation of $\mathrm{AdS}_{5} / \mathrm{SYM}_{4}$ duality, where $\mathrm{SYM}$ becomes free at a special point $\left(g_{\mathrm{YM}}=0\right)$ in the moduli space, but in order for SYM to precisely correspond to the supergravity side one has to turn on the coupling $g_{\mathrm{YM}}$. The orbifold point is the analogue of the free SYM. In the following, we will consider the orbifold point of the D1-D5 CFT.

\section{A.2. Orbifold CFT}

The $\mathcal{N}=(4,4)$ SCFT at the orbifold point $\mathcal{M}_{0}=\left(T^{4}\right)^{N} / S_{N}$ is described by the free Lagrangian

$$
S=\frac{1}{2 \pi} \int \mathrm{d}^{2} \sigma\left[\partial x_{A}^{a} \bar{\partial} x_{A}^{a}+\psi_{A}^{a}(z) \bar{\partial} \psi_{A}^{a}(z)+\widetilde{\psi}_{A}^{a}(\bar{z}) \partial \widetilde{\psi}_{A}^{a}(\bar{z})\right]
$$

where $a=6,7,8,9$ labels the $T^{4}$ directions and $A=1, \ldots, N$ labels the $N$ copies of $T^{4}$. Summation over $a$ and $A$ is implied. Without the orbifolding, this theory would be simply a direct sum of $N$ free CFTs each with $c=6$.

As we explained in the last subsection, this theory has $S O(4)_{E} \cong S U(2)_{R} \times \widetilde{S U(2)_{R}}$ $R$-symmetry and $S O(4)_{I} \cong S U(2)_{I} \times \widetilde{S U(2)}_{I}$ non- $R$-symmetry. The transformation property of the fields under these symmetry groups is as follows ${ }^{8}$ :

\begin{tabular}{|c|c|c|}
\hline Field & $S U(2)_{R} \times \widetilde{S U(2)_{R}}$ & $S U(2)_{I} \times \widetilde{S U(2)_{I}}$ \\
\hline$x^{a}$ & $(\mathbf{1}, \mathbf{1})$ & $(\mathbf{2}, \mathbf{2})$ \\
\hline$\psi^{a}$ & $(\mathbf{2}, \mathbf{1})$ & $(\mathbf{1}, \mathbf{2})$ \\
\hline$\widetilde{\psi}^{a}$ & $(\mathbf{1}, \mathbf{2})$ & $(\mathbf{1}, \mathbf{2})$ \\
\hline
\end{tabular}

Following [66], we bosonize the fermions as

$\Psi_{A}^{+}(z) \equiv \frac{1}{\sqrt{2}}\left(\psi_{A}^{1}+\mathrm{i} \psi_{A}^{2}\right)=\mathrm{e}^{\mathrm{i} \phi_{A}^{5}(z)}, \quad \Psi_{A}^{-}(z) \equiv \frac{1}{\sqrt{2}}\left(\psi_{A}^{3}+\mathrm{i} \psi_{A}^{4}\right)=\mathrm{e}^{\mathrm{i} \phi_{A}^{6}(z)}$,

8 The surviving supersymmetry is in the representation $\left(+\frac{1}{2} ; \mathbf{2}, \mathbf{1} ; \mathbf{2}, \mathbf{1}\right)$ and $\left(-\frac{1}{2} ; \mathbf{1}, \mathbf{2} ; \mathbf{2}, \mathbf{1}\right)$ under $S O(1,1)_{05} \times$ $\left[S U(2)_{R} \times \widehat{S U(2)_{R}}\right] \times\left[S U(2)_{I} \times \widehat{S U(2)_{I}}\right]$. This implies the transformation property (A.3) of the hypermultiplet superpartners of the boson $x^{a}$ (see, e.g., [70]). 
where left-moving bosons are normalized as $\phi^{i}\left(z_{1}\right) \phi^{j}\left(z_{2}\right) \sim-\delta^{i j} \log \left(z_{1}-z_{2}\right)$. Similarly, the right-moving fermions $\widetilde{\Psi}_{A}^{ \pm}(\bar{z})$ are bosonized using right-moving bosons $\widetilde{\phi}_{A}^{i}(\bar{z})$. In terms of bosons, the $R$ current is

$$
J_{R}^{3}(z)=\frac{\mathrm{i}}{2} \sum_{A=1}^{N}\left(\partial \phi_{A}^{5}-\partial \phi_{A}^{6}\right)(z), \quad J_{R}^{ \pm}(z)=\sum_{A=1}^{N} \mathrm{e}^{ \pm \mathrm{i}\left(\phi_{A}^{5}-\phi_{A}^{6}\right)(z)} .
$$

Note that $\Psi_{A}^{s}(z), s= \pm$ have $R$-charge $J_{R}^{3}=\frac{s}{2}$, while $\widetilde{\Psi}_{A}^{\widetilde{s}}(\bar{z}), \widetilde{s}= \pm$ have $\widetilde{J}_{R}^{3}=\frac{\widetilde{s}}{2}$.

The charge associated with the global $\widehat{S U(2)_{I}}$ symmetry is given by

$$
\widetilde{I}^{i}=\widetilde{I}_{\mathrm{hol}}^{i}+\widetilde{I}_{\mathrm{antihol}}^{i},
$$

where

$$
\widetilde{I}_{\text {hol }}^{i}=\int \frac{\mathrm{d} z}{2 \pi \mathrm{i}} \widetilde{I}_{\text {hol }}^{i}(z), \quad \widetilde{I}_{\text {antihol }}^{i}=\int \frac{\mathrm{d} \bar{z}}{2 \pi i} \widetilde{I}_{\text {antihol }}^{i}(\bar{z}) .
$$

The currents $\widetilde{I}_{\text {hol }}^{i}(z), \widetilde{I}_{\text {antihol }}^{i}(\bar{z})$ are given by

$$
\begin{array}{ll}
\widetilde{I}_{\text {hol }}^{3}(z)=\frac{\mathrm{i}}{2} \sum_{A=1}^{N}\left(\partial \phi_{A}^{5}+\partial \phi_{A}^{6}\right)(z), & \widetilde{I}_{\text {hol }}^{ \pm}(z)=\sum_{A=1}^{N} \mathrm{e}^{ \pm \mathrm{i}\left(\phi_{A}^{5}+\phi_{A}^{6}\right)(z)}, \\
\widetilde{I}_{\text {antihol }}^{3}(\bar{z})=\frac{\mathrm{i}}{2} \sum_{A=1}^{N}\left(\bar{\partial} \widetilde{\phi}_{A}^{5}+\bar{\partial} \widetilde{\phi}_{A}^{6}\right)(\bar{z}), & \widetilde{I}_{\text {antihol }}^{ \pm}(\bar{z})=\sum_{A=1}^{N} \mathrm{e}^{ \pm \mathrm{i}\left(\widetilde{\phi}_{A}^{5}+\widetilde{\phi}_{A}^{6}\right)(\bar{z})},
\end{array}
$$

where we omitted the part that contains $x^{a}$ fields only, which is not relevant for us; see [68] for the complete expression. The charge $I^{i}$ associated with the global $S U(2)_{I}$ does not involve fermions and can also be found in [68]. Note that holomorphic part $\widetilde{I}_{\text {hol }}^{i}$ and the antiholomorphic part $\widetilde{I}_{\text {antihol }}^{i}$ are not separately conserved; the chirality of the CFT fields is not aligned with the chirality of the $S O(4)_{I} \cong S U(2)_{I} \times \widehat{S U(2)_{I}}$ symmetry.

It turns out to be convenient to define $\Phi_{A}^{\widetilde{\alpha}}(z)$ by

$$
\begin{aligned}
& \Phi_{A}^{+}(z)=\Psi_{A}^{+}(z)=\frac{1}{\sqrt{2}}\left(\psi_{A}^{1}+\mathrm{i} \psi_{A}^{2}\right)=\mathrm{e}^{\mathrm{i} \phi_{A}^{5}(z)}, \\
& \Phi_{A}^{-}(z)=\Psi_{A}^{-}(z)^{\dagger}=\frac{1}{\sqrt{2}}\left(\psi_{A}^{3}-\mathrm{i} \psi_{A}^{4}\right)=\mathrm{e}^{-\mathrm{i} \phi_{A}^{6}(z)} .
\end{aligned}
$$

We similarly define $\widetilde{\Phi}_{A}^{\widetilde{\alpha}}(\bar{z})$. Note that $\Phi_{A}^{\widetilde{\alpha}}(z)$ and $\widetilde{\Phi}_{A}^{\widetilde{\alpha}}(\bar{z})$ have $\widetilde{I}$-charge $\widetilde{I}^{3}=\frac{\widetilde{\alpha}}{2}$.

In the $S_{N}$ orbifold CFT (A.2), there are twist fields $\sigma_{P}(z), P \in S_{N}$, which permute the copies of CFT as $1 \rightarrow P(1), 2 \rightarrow P(2), \ldots, N \rightarrow P(N)$ as one circles the point of insertion of $\sigma_{P}$ [58]. For example, if we have $\sigma_{(12, \ldots, n)}(z)$ at $z=0$, we should impose the boundary condition on the fields $x_{A}(z), \psi_{A}(z)$ as follows,

$$
\begin{aligned}
& x_{1}\left(\mathrm{e}^{2 \pi \mathrm{i}} z\right)=x_{2}(z), \ldots, x_{n}\left(\mathrm{e}^{2 \pi \mathrm{i}} z\right)=x_{1}(z), \\
& \psi_{1}\left(\mathrm{e}^{2 \pi \mathrm{i}} z\right)= \pm \psi_{2}(z), \ldots, \psi_{n}\left(\mathrm{e}^{2 \pi \mathrm{i}} z\right)= \pm \psi_{1}(z),
\end{aligned}
$$

where ' + ' is for the NS sector and ' - ' is for the R sector. This permutation of CFTs can be conveniently realized by going to a covering space on which the fields of the CFT are single valued $[64,66]$. In the case of $\sigma_{(12, \ldots, n)}(z=0)$, one can define a new coordinate $t$ by

$$
t^{n}=b z \quad \text { near } \quad z=0,
$$

so that circling $n$ times around $z=0$ corresponds to circling around $t=0$ once. This corresponds to inserting a twist field at $z=0$ in the $z$-space that has the lowest conformal weight $\Delta_{n}=\frac{1}{4}\left(n-\frac{1}{n}\right)[64,66]$. We will denote this twist operator henceforth by $\sigma_{n}(z)$. Twist fields with higher conformal weight are obtained by inserting some fields at $t=0$ in the $t$-space. In this way, computing the correlation function of twist fields reduces to finding 
a holomorphic map between the $z$-space and the covering $t$-space that realizes the twists $[64,66]$. Note that this method of covering space is applicable only to $S_{N}$ orbifolds, and not applicable to general non-Abelian orbifolds [71].

One example of the operators that can be obtained by inserting a field at $t=0$ is the chiral primary operator $\sigma_{n}^{--}(z)$ in the NS sector. Concretely, $\sigma_{n}^{--}(z)$ is obtained by inserting in the $t$-space the following operator [66],

$$
\sigma_{n}^{--}(t) \equiv \sigma_{n}^{-}(t) \widetilde{\sigma}_{n}^{-}(\bar{t}),
$$

where

$$
\sigma_{n}^{-}(t) \equiv b^{-p^{2} / n} \mathrm{e}^{\mathrm{i} p\left(\phi^{5}-\phi^{6}\right)}(t), \quad \widetilde{\sigma}_{n}^{-}(\bar{t}) \equiv \bar{b}^{-p^{2} / n} \mathrm{e}^{\mathrm{i} p\left(\widetilde{\phi}^{5}-\widetilde{\phi}^{6}\right)}(\bar{t}) .
$$

Here $p \equiv \frac{n-1}{2}$, and $\phi^{i}(t), \widetilde{\phi}^{i}(\bar{t})$ are the lift of $\phi_{A}^{i}(z), \widetilde{\phi}_{A}^{i}(\bar{z})$ to the $t$-space, whose OPE is $\phi^{i}\left(t_{1}\right) \phi^{j}\left(t_{2}\right) \sim-\log \left(t_{1}-t_{2}\right)$. Note that $\sigma_{n}^{--}(z)$ is not the coordinate transformation of $\sigma_{n}^{--}(t)$, but it is a 'product' of the pure twist operator $\sigma_{n}(z)$ and the insertion $\sigma_{n}^{--}(t)$. Therefore, the conformal dimension of $\sigma_{n}^{--}(z)$ is given by

$$
h=\Delta_{n}+\frac{1}{n}\left(\frac{p^{2}}{2}+\frac{p^{2}}{2}\right)=\frac{n-1}{2} .
$$

Here $\Delta_{n}=\frac{1}{4}\left(n-\frac{1}{n}\right)$ is the conformal dimension of the pure twist $\sigma_{n}(z)$. In the second term, we divided the conformal dimension in the $t$-space by $n$ to obtain the conformal dimension in the $z$-space (remember that $z \propto t^{n}$ ) [66]. Similarly one can show that $(h, \tilde{h})=\left(j_{R}^{3}, \widetilde{j}_{R}^{3}\right)=\left(\frac{n-1}{2}, \frac{n-1}{2}\right)$. The chiral primary $\sigma_{n}^{--}(z)$ has the smallest conformal dimension among the chiral primary operators constructed on $\sigma_{n}(z)$ [66]. Another important fact is that $\sigma_{1}^{--}(z)$ is nothing but the unit operator.

The twist fields $\sigma_{P}(z), P \in S_{N}$, considered above are not proper fields of the orbifold CFT. A proper field of the $S_{N}$ orbifold CFT should be invariant under conjugation by any element of $S_{N}$. This means that the twist sector is in one-to-one correspondence with the conjugacy class of $S_{N}$ [71]. One can construct a proper field from $\sigma_{P}(z)$ by

$$
\Sigma_{P}(z)=\frac{\lambda_{P}}{N !} \sum_{Q \in S_{N}} \sigma_{Q P Q^{-1}}(z),
$$

where $\lambda_{P}$ is a normalization constant. As long as we do this summation over $S_{N}$ at the end of the computation of the correlation function, we can consider the cyclic permutation $\sigma_{(12, \ldots, n)}(z) \equiv \sigma_{n}(z)$, instead of $\sigma_{P}(z)$ with general $P \in S_{N}$.

Let us mention here one important aspect of the covering space method. As we discussed above, computing correlation functions of twist fields reduces to the problem of finding a holomorphic map $z=f(t)$ that realizes the twists. This coordinate transformation leads to a nontrivial Liouville action $S_{L}[f(t)]$, which contributes to the correlation function as $\mathrm{e}^{S_{L}[f(t)]}$ $[64,66]$. This Liouville factor is important when, for example, computing the correlation function of twist fields at $z=z_{i}$; the precise form of the map $f(t)$ depends on $z_{i}$, and this in turn leads to a nontrivial dependence of the correlation function on $z_{i}-z_{j}$. Actually the normalization in (A.13), which depends also on the map $f(t)$, also gives a nontrivial contribution. However, what we will be interested in here is the $z_{i}$ dependence of the correlation functions of non-twist operators at general points $z=z_{i}$ and twist operators at fixed points $z=0, \infty$. Because $\mathrm{e}^{S_{L}}$ and the normalization of twist operators depend only on the coordinates of twist operators, they are irrelevant for us and we will ignore them altogether. 
Table 2. Single-trace chiral primaries in the NS sector. Here $n=1,2, \ldots, N$, and $\widetilde{\alpha}, \widetilde{\beta}= \pm$. Summation over $A=1,2, \ldots, N$ is implied in all expressions, even if $A$ appears only once (i.e., $\left.\Phi_{A}^{\tilde{\alpha}}=\sum_{A=1}^{N} \Phi_{A}^{\tilde{\alpha}}\right)$. Note that $\sigma_{1}^{--}=1$. The corresponding cohomology $H^{2 h, 2 \tilde{h}}(B)$, and the weights and charge of the chiral primary field are shown. The $R$-charge of the corresponding $\mathrm{R}$ ground state is also shown.

\begin{tabular}{|c|c|c|c|c|}
\hline Chiral primaries & $H^{2 h, 2 \tilde{h}}(B)$ & $(h, \tilde{h})_{\mathrm{NS}}=\left(j_{R}^{3}, \widetilde{j}_{R}^{3}\right)_{\mathrm{NS}}$ & $S U(2)_{I} \times \widetilde{S U(2)_{I}}$ & $\left(j_{R}^{3}, \widetilde{j}_{R}^{3}\right)_{\mathrm{R}}$ \\
\hline$\overline{\sigma_{n}^{--}}$ & $H^{0,0}$ & $\left(\frac{n-1}{2}, \frac{n-1}{2}\right)$ & $(1,1)$ & $\left(-\frac{1}{2},-\frac{1}{2}\right)$ \\
\hline$\tau_{n}^{\widetilde{\alpha}, \widetilde{s}=-}=\Phi_{A}^{\widetilde{\alpha}} \sigma_{n}^{--}$ & $H^{1,0}$ & $\left(\frac{n}{2}, \frac{n-1}{2}\right)$ & $(1,2)$ & $\left(0,-\frac{1}{2}\right)$ \\
\hline$\tau_{n}^{s=-, \widetilde{\alpha}}=\widetilde{\Phi}_{A}^{\widetilde{\alpha}} \sigma_{n}^{--}$ & $H^{0,1}$ & $\left(\frac{n-1}{2}, \frac{n}{2}\right)$ & $(1,2)$ & $\left(-\frac{1}{2}, 0\right)$ \\
\hline$\sigma_{n}^{+-}=\Phi_{A}^{+} \Phi_{A}^{-} \sigma_{n}^{--}$ & $H^{2,0}$ & $\left(\frac{n+1}{2}, \frac{n-1}{2}\right)$ & $(1,1)$ & $\left(\frac{1}{2},-\frac{1}{2}\right)$ \\
\hline$\sigma_{n}^{\widetilde{\alpha} \widetilde{\beta}}=\Phi_{A}^{\widetilde{\alpha}} \widetilde{\Phi}_{A}^{\widetilde{\beta}} \sigma_{n}^{--}$ & $H^{1,1}$ & $\left(\frac{n}{2}, \frac{n}{2}\right)$ & $(1,3) \oplus(1,1)$ & $(0,0)$ \\
\hline$\sigma_{n}^{-+}=\widetilde{\Phi}_{A}^{+} \widetilde{\Phi}_{A}^{-} \sigma_{n}^{--}$ & $H^{0,2}$ & $\left(\frac{n-1}{2}, \frac{n+1}{2}\right)$ & $(1,1)$ & $\left(-\frac{1}{2}, \frac{1}{2}\right)$ \\
\hline$\tau_{n}^{s=+, \widetilde{\alpha}}=\Phi_{A}^{+} \Phi_{A}^{-} \widetilde{\Phi}_{A}^{\widetilde{\alpha}} \sigma_{n}^{--}$ & $H^{2,1}$ & $\left(\frac{n+1}{2}, \frac{n}{2}\right)$ & $(1,2)$ & $\left(\frac{1}{2}, 0\right)$ \\
\hline$\tau_{n}^{\tilde{\alpha}, \widetilde{s}=+}=\Phi_{A}^{\widetilde{\alpha}} \widetilde{\Phi}_{A}^{+} \widetilde{\Phi}_{A}^{-} \sigma_{n}^{--}$ & $H^{1,2}$ & $\left(\frac{n}{2}, \frac{n+1}{2}\right)$ & $(1,2)$ & $\left(0, \frac{1}{2}\right)$ \\
\hline$\sigma_{n}^{++}=\Phi_{A}^{+} \Phi_{A}^{-} \widetilde{\Phi}_{A}^{+} \widetilde{\Phi}_{A}^{-} \sigma_{n}^{--}$ & $H^{2,2}$ & $\left(\frac{n+1}{2}, \frac{n+1}{2}\right)$ & $(1,1)$ & $\left(\frac{1}{2}, \frac{1}{2}\right)$ \\
\hline
\end{tabular}

\section{A.3. Chiral primaries and spectral flow to $R$ sector}

We are interested in the R sector ground states of the D1-D5 CFT. The R ground states can be obtained by first finding chiral primary operators in the NS sector, and then spectral flowing to the $\mathrm{R}$ sector.

So, let us first focus on the chiral primaries of the orbifold CFT (A.2) in the NS sector. The chiral primaries with weight $(h, \tilde{h})$ of $\mathcal{N}=(4,4)$ SCFT on a manifold $K$ correspond to the elements of the cohomology $H^{2 h, 2 \tilde{h}}(K)$ [72]. In the present case of the orbifold $K=\left(T^{4}\right)^{N} / S_{N}$, the cohomology $H^{*}(K)$ can be constructed as follows [73]. Let the basis of $H^{*}(B)$ be $w^{a}, a=1,2, \ldots, \operatorname{dim}\left(H^{*}(B)\right)=16$, where $B$ is the diagonal $T^{4}$, i.e., the sum of all copies of $T^{4}$. For each $w^{a}$, introduce a 'one-particle creation operator' $\alpha_{-n}^{a}, n=1,2, \ldots$ Then there is a one-to-one correspondence between the elements of $H^{*}(K)$ and the states in the 'Fock space' generated by $\alpha_{-n}^{a}$. Namely, for each element of $H^{*}(K)$, there is a state $\prod_{n, a}\left(\alpha_{-n}^{a}\right)^{N_{n a}}|0\rangle, \sum_{n} n N_{n}=N$. If $w^{a}$ is an even (odd) form, $\alpha_{-n}^{a}$ is bosonic (fermionic).

In the present case, $H^{*}(B)$ has eight elements of even rank and eight elements of odd rank, so there are corresponding $8+8$ chiral primaries. The chiral primaries corresponding to $\alpha_{-n}^{a}$ are constructed by multiplying the above $8+8$ chiral primaries with the chiral primary $\sigma_{n}^{--}(z)$ defined in (A.12), which is a twist operator of order $n$. In table 2, we list all chiral primary fields in the NS sector that correspond to the 'one-particle creation operator' $\alpha_{-n}^{a}$. They are single-trace in the sense that they involve only one summation over copies $\sum_{A=1}^{N}$ and only one twist operator $\sigma_{n}^{--}(z)$. We also present their conformal weight, $R$-charges, and $S U(2)_{I} \times \widetilde{S U(2)_{I}}$ charges, as well as the $R$-charges of the $\mathrm{R}$ ground states that can be obtained by spectral flow, using (A.18). One sees that there are eight bosonic and eight fermionic single-trace chiral primaries:

$$
\sigma_{n}^{s \widetilde{s}}, \sigma_{n}^{\widetilde{\alpha} \widetilde{\beta}}, \tau_{n}^{s \widetilde{\alpha}}, \tau_{n}^{\widetilde{\alpha s}}
$$

Here, $s, \widetilde{s}= \pm$ correspond to $S U(2)_{R} \times \widetilde{S U(2)}_{R}$ charges $\left(J_{R}^{3}, \widetilde{J}_{R}^{3}\right)=\left(\frac{s}{2}, \frac{\widetilde{s}}{2}\right)$, while $\widetilde{\alpha}, \widetilde{\beta}= \pm$ correspond to $\widetilde{S U(2)_{I}}$ charge $\widetilde{I}^{3}=\frac{\widetilde{\alpha}}{2}$. The fields $\tau_{n}^{s \widetilde{\alpha}}, \tau_{n}^{\widetilde{\alpha s}}$ which correspond to odd-rank elements of $H^{*}(B)$ are indeed fermionic because $\Phi$ anticommute. These single-trace chiral 
primaries are known to be in one-to-one correspondence with the Kaluza-Klein spectrum of particle supergravity on $\mathrm{AdS}_{3} \times S^{3} \times T^{4}$ [40]. We will use $\sigma_{n}^{--}, \sigma_{n}^{++}$etc, with explicit +, signs exclusively for denoting the $\sigma_{n}^{s \widetilde{s}}$ operators, not $\sigma_{n}^{\widetilde{\alpha} \widetilde{\beta}}$.

Now let us consider spectral flowing to the R sector. From (A.5), the spectral flow operator that maps NS sector operators to the R sector operators is

$$
U(z)=\exp \left[-\frac{\mathrm{i}}{2} \sum_{A=1}^{N}\left(\phi_{A}^{5}-\phi_{A}^{6}\right)\right](z),
$$

where we wrote the holomorphic part only. The spectral flow relates the charges in the R and NS sectors as follows:

$$
h_{\mathrm{R}}=h_{\mathrm{NS}}-\left(j_{R}^{3}\right)_{\mathrm{NS}}+\frac{c}{24}, \quad\left(j_{R}^{3}\right)_{\mathrm{R}}=\left(j_{R}^{3}\right)_{\mathrm{NS}}-\frac{c}{12} .
$$

Here, roman $\mathrm{R}$ stands for Ramond, while italic $R$ is for $R$-charge. The spectral flow operator in the $t$-space is given by coordinate transformation of $U(z)$ by

$$
U(t=0) \propto \exp \left[-i \frac{n}{2}\left(\phi^{5}-\phi^{6}\right)\right](t=0),
$$

where we used $\phi_{A}(z) \rightarrow \phi\left(t \mathrm{e}^{2 \pi \mathrm{i}(A-1) / n}\right) \underset{t \rightarrow 0}{\longrightarrow} \phi(t=0)$. For example, we can use this to map the NS sector twist operator $\left[\sigma_{n}^{-}(t)\right]_{\mathrm{NS}} \propto \exp \left[\mathrm{i} \frac{n-1}{2}\left(\phi^{5}-\phi^{6}\right)\right](t)$ into the R sector:

$$
\left[\sigma_{n}^{-}(t)\right]_{\mathrm{NS}} \rightarrow\left[\sigma_{n}^{-}(t)\right]_{\mathrm{R}} \propto \mathrm{e}^{-\frac{\mathrm{i}}{2}\left(\phi^{5}-\phi^{6}\right)}(t) .
$$

We can check that this has the correct conformal dimension and $R$-charge as a $\mathrm{R}$ ground state:

$h_{\mathrm{R}}=\Delta_{n}+\frac{1}{n}\left[\frac{1}{2}\left(\frac{1}{2}\right)^{2}+\frac{1}{2}\left(\frac{1}{2}\right)^{2}\right]=\frac{n}{4}=\frac{c}{24}, \quad\left(j_{R}^{3}\right)_{\mathrm{R}}=-\frac{1}{2}$.

Including other operators, the list of R ground states corresponding to single-trace NS chiral primaries is

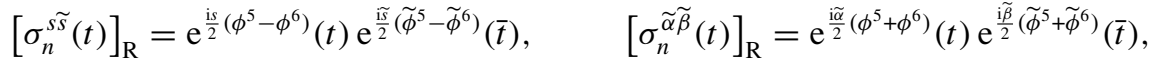

$$
\begin{aligned}
& {\left[\tau_{n}^{s \widetilde{\alpha}}(t)\right]_{\mathrm{R}}=\mathrm{e}^{\frac{\mathrm{i} s}{2}\left(\phi^{5}-\phi^{6}\right)}(t) \mathrm{e}^{\frac{\mathrm{i} \widetilde{\alpha}}{2}\left(\widetilde{\phi}^{5}+\tilde{\phi}^{6}\right)}(\bar{t}), \quad\left[\tau_{n}^{\widetilde{\alpha} \widetilde{s}}(t)\right]_{\mathrm{R}}=\mathrm{e}^{\frac{\mathrm{i} \tilde{\alpha}}{2}\left(\phi^{5}+\phi^{6}\right)}(t) \mathrm{e}^{\left.\frac{\tilde{\tilde{s}}}{2} \widetilde{\phi}^{5}-\widetilde{\phi}^{6}\right)}(\bar{t}) .}
\end{aligned}
$$

Here, we ignored normalization constants because they are irrelevant for our purposes as explained at the end of the last subsection. We will call these operators (A.22) single-trace $\mathrm{R}$ ground states. Henceforth, we restrict ourselves to the $\mathrm{R}$ sector and drop the subscript $\mathrm{R}$ from the twist operators (A.22).

General $\mathrm{R}$ ground states are obtained by multiplying the single-trace $\mathrm{R}$ ground states (A.22) together. They can be written as

$$
\begin{aligned}
& \sigma=\prod_{n, \mu}\left(\sigma_{n}^{\mu}\right)^{N_{n \mu}}\left(\tau_{n}^{\mu}\right)^{N_{n \mu}^{\prime}}, \\
& \sum_{n, \mu} n\left(N_{n \mu}+N_{n \mu}^{\prime}\right)=N, \quad N_{n \mu}=0,1,2, \ldots, \quad N_{n \mu}^{\prime}=0,1,
\end{aligned}
$$

where $\mu$ labels the eight polarizations of bosons and fermions, i.e., $\mu=(s, \widetilde{s}),(\widetilde{\alpha}, \widetilde{\beta})$ for bosons $\mu=(s, \widetilde{\alpha}),(\widetilde{\alpha}, \widetilde{s})$ for fermions. The numbers

$$
\left\{N_{n \mu}, N_{n \mu}^{\prime}\right\}
$$

uniquely specify the $\mathrm{R}$ ground state. We will refer to the factors $\sigma_{n}^{\mu}, \tau_{n}^{\mu}$ in (A.23) as constituent twist operators of the twist operator $\sigma$. 


\section{A.4. Correlation function of non-twist operators}

We would now like compute correlation functions in the R ground states of the D1-D5 CFT. Such CFT correlation functions are related to supergravity amplitudes in the dual geometry via AdS/CFT.

We want to compute the two-point function of the 'probe' operator $\mathcal{A}$ in the state created by a general twist operator. Let us assume that $\mathcal{A}$ does not contain twists and can be written as a sum over copies of the CFT,

$$
\mathcal{A}=\frac{1}{\sqrt{N}} \sum_{A=1}^{N} \mathcal{A}_{A}
$$

where $\mathcal{A}_{A}$ is a non-twist operator that lives in the Ath copy. For example, we can take

$$
\mathcal{A}_{A}=\partial X_{A}^{a}(z) \bar{\partial} X_{A}^{b}(\bar{z})
$$

which corresponds to fluctuation of the metric in the internal $T^{4}$ direction.

Let us consider the general $\mathrm{R}$ ground state (A.23). If we denote $\sigma_{n}^{\mu}, \tau_{n}^{\mu}$ collectively by $\sigma_{n}^{\widehat{\mu}}$, and $N_{n \mu}, N_{n \mu}^{\prime}$ by $N_{n \widehat{\mu}}$, then we can write (A.23) as

$$
\sigma=\prod_{n, \widehat{\mu}}\left(\sigma_{n}^{\widehat{\mu}}\right)^{N_{n \widehat{\mu}}} .
$$

The correlation function of the probe operator $\mathcal{A}$ in this state is, taking into account the summation over copies (equation (A.15)),

$$
\begin{aligned}
\left\langle\Sigma^{\dagger} \mathcal{A}^{\dagger} \mathcal{A} \Sigma\right\rangle & =\left\langle\sigma^{\dagger} \mathcal{A}^{\dagger} \mathcal{A} \sigma\right\rangle=\frac{1}{N} \sum_{A, B=1}^{N}\left\langle\sigma^{\dagger} \mathcal{A}_{A}^{\dagger} \mathcal{A}_{B} \sigma\right\rangle \\
& =\frac{1}{N} \sum_{A, B=1}^{N}\left\langle\left[\prod_{n, \widehat{\mu}}\left(\sigma_{n}^{\widehat{\mu}}\right)^{N_{n \widehat{\mu}}}\right]^{\dagger} \mathcal{A}_{A}^{\dagger} \mathcal{A}_{B}\left[\prod_{v, \widehat{\mu}}\left(\sigma_{n}^{\widehat{\mu}}\right)^{N_{n \widehat{\mu}}}\right]\right\rangle \\
& =\frac{1}{N} \sum_{n, \widehat{\mu}} N_{n \widehat{\mu}} \sum_{A, B \in \sigma_{n}^{\widehat{\mu}}}\left\langle\left[\sigma_{n}^{\widehat{\mu}}\right]^{\dagger} \mathcal{A}_{A}^{\dagger} \mathcal{A}_{B} \sigma_{n}^{\widehat{\mu}}\right\rangle \\
& =\frac{1}{N} \sum_{n, \widehat{\mu}} n N_{n \widehat{\mu}} \sum_{A=1}^{n}\left\langle\left[\sigma_{(1, \ldots, n)}^{\widehat{\mu}}\right]^{\dagger} \mathcal{A}_{A}^{\dagger} \mathcal{A}_{1} \sigma_{(1, \ldots, n)}^{\widehat{\mu}}\right\rangle,
\end{aligned}
$$

where $\sum_{A, B \in \sigma_{n}^{\widehat{\mu}}}$ means summing over copies $A, B$ that are involved in the $n$-cycle of $\sigma_{n}^{\widehat{\mu}}$. In the first equality, we used the fact that $\mathcal{A}$ is a sum over copies, (A.25). In the fourth equality, we used the fact that the 'initial' and 'final' states must have the same length of twist and the same $S U$ (2) charges to give a non-vanishing correlator, since $\mathcal{A}_{A}^{\dagger} \mathcal{A}_{B}$ does not involve twist or charges. We assumed that the three-point function vanishes, $\left\langle\left[\sigma_{n}^{\widehat{\mu}}\right]^{\dagger} \mathcal{A}_{A} \sigma_{n}^{\widehat{\mu}}\right\rangle=0$, which is true in the case considered in this paper. Note that the final expression (A.28) decomposed into contributions from constituent twist operators. This is because we restrict ourselves to non-twist probes $\mathcal{A}$, and because we are in the orbifold point approximation and ignoring interactions. Once we start considering twist probes or interaction, this will no longer be the case.

Therefore, for a non-twist operator $\mathcal{A}$ in the orbifold approximation, all we have to compute is the four-point function

$\left\langle\left[\sigma_{(1, \ldots, n)}^{\widehat{\mu}}(z=\infty)\right]^{\dagger} \mathcal{A}_{A}\left(z_{1}\right)^{\dagger} \mathcal{A}_{B}\left(z_{2}\right) \sigma_{(1, \ldots, n)}^{\widehat{\mu}}(z=0)\right\rangle \equiv\left\langle\mathcal{A}_{A}\left(z_{1}\right)^{\dagger} \mathcal{A}_{B}\left(z_{2}\right)\right\rangle_{\sigma_{(1, \ldots, n)}^{\widehat{\mu}}}$,

where $1 \leqslant A, B \leqslant n$. 


\section{A.5. Boson correlation function}

Let us evaluate the correlation function (A.28), (A.29) for $\mathcal{A}$ a purely bosonic non-twist operator such as $\partial X \bar{\partial} X$. In this case, we can replace the twist operator $\sigma_{(1, \ldots, n)}^{\widehat{\mu}}(z)$ in (A.29) with the pure twist operator $\sigma_{(1, \ldots, n)}(z)$, since these two are different only in their fermionic dressing, to which the bosonic operator $\mathcal{A}$ is insensitive.

The twist operators $\sigma_{(1, \ldots, n)}(z)$ at $z=0, \infty$ mean that $X_{A}^{a}(z)$ permute as $X_{1}^{a} \rightarrow X_{2}^{a} \rightarrow$ $\cdots \rightarrow X_{n}^{a} \rightarrow X_{1}^{a}$ as one circles $z=0, \infty$. As explained around (A.11), we can conveniently go to the covering $t$-space by

$$
z=b t^{n}
$$

on which we have single-valued fields $X^{a}(t)$. If we normalize the correlation function in the $t$-space as

$$
\left\langle\mathcal{A}_{A}^{\dagger}\left(t_{1}\right) \mathcal{A}_{B}\left(t_{2}\right)\right\rangle=\frac{C}{\left(t_{1}-t_{2}\right)^{2 h}\left(\bar{t}_{1}-\bar{t}_{2}\right)^{2 \tilde{h}}},
$$

where $(h, \tilde{h})$ is the conformal weight of $\mathcal{A}$, then the correlation function on the $z$-plane is

$$
\left\langle\mathcal{A}_{A}^{\dagger}\left(z_{1}\right) \mathcal{A}_{B}\left(z_{2}\right)\right\rangle_{\sigma_{(1, \ldots, n)}}=\frac{C}{n^{2 h+2 \tilde{h}}\left(z_{1} z_{2}\right)^{h}\left(\bar{z}_{1} \bar{z}_{2}\right)^{\tilde{h}}\left[\left(\frac{z_{1}}{z_{2}}\right)^{\frac{1}{2 n}}-\left(\frac{z_{2}}{z_{1}}\right)^{\frac{1}{2 n}}\right]^{2 h}\left[\left(\frac{\bar{z}_{1}}{\bar{z}_{2}}\right)^{\frac{1}{2 n}}-\left(\frac{\bar{z}_{2}}{\bar{z}_{1}}\right)^{\frac{1}{2 n}}\right]^{2 \tilde{h}}} .
$$

If we go to the cylinder coordinate $w$ by

$$
z=\mathrm{e}^{-\mathrm{i} w}
$$

then the correlation function is

$$
\left\langle\mathcal{A}_{A}^{\dagger}\left(w_{1}\right) \mathcal{A}_{B}\left(w_{2}\right)\right\rangle_{\sigma_{(1, \ldots, n)}}=\frac{C}{\left[2 n \sin \left(\frac{w}{2 n}\right)\right]^{2 h}\left[2 n \sin \left(\frac{\bar{w}}{2 n}\right)\right]^{2 \tilde{h}}},
$$

where

$$
w \equiv w_{1}-w_{2}, \quad \bar{w} \equiv \bar{w}_{1}-\bar{w}_{2} .
$$

Here, the copy labels $A, B$ mean that $w_{1}$ and $w_{2}$ must be understood as $w_{1}+2 \pi(A-1)$ and $w_{2}+2 \pi(B-1)$, respectively.

The result (A.33) expresses the fact that the effective circumference of the CFT cylinder is $2 \pi n$, where the factor of $n$ comes from the permutation of $n$ copies of the CFT. Indeed, from this picture one can easily write down (A.33) directly, simply by inserting the appropriate factors of $n$ in the usual free correlator on the cylinder.

\section{A.6. Fermion correlation function}

Now let us evaluate the correlation function (A.29) in the case where $\mathcal{A}$ involves fermions $\psi$. As an example, let us consider

$$
G_{z} \equiv\left\langle\sigma_{n}^{--}\left(z_{\infty}\right)^{\dagger} \Psi_{A}^{+}\left(z_{1}\right)^{\dagger} \Psi_{B}^{+}\left(z_{2}\right) \sigma_{n}^{--}\left(z_{0}\right)\right\rangle .
$$

It is understood that we will take $z_{\infty} \rightarrow \infty, z_{0} \rightarrow 0$ in the end, so we can use (A.30) as the relation between $z$ and $t$ coordinates. Using the expression of operators in terms of bosons (equations (A.4), (A.22)), one computes

$$
\begin{aligned}
G_{z} \propto \mathrm{e}^{S_{L}}\left\langle\left[\mathrm{e}^{\frac{\mathrm{i}}{2}\left(\phi^{5}-\phi^{6}\right)}\left(t_{\infty}\right) \mathrm{e}^{-\frac{\mathrm{i}}{2}\left(\widetilde{\phi}^{5}-\widetilde{\phi}^{6}\right)}\left(\bar{t}_{\infty}\right)\right] \mathrm{e}^{-\mathrm{i} \phi^{5}}\left(t_{1}\right) \mathrm{e}^{\mathrm{i} \phi^{5}}\left(t_{2}\right)\left[\mathrm{e}^{-\frac{\mathrm{i}}{2}\left(\phi^{5}-\phi^{6}\right)}\left(t_{0}\right) \mathrm{e}^{\frac{\mathrm{i}}{2}\left(\widetilde{\phi}^{5}-\widetilde{\phi}^{6}\right)}\left(\bar{t}_{0}\right)\right]\right\rangle \\
\times\left(\frac{\mathrm{d} t_{1}}{\mathrm{~d} z_{1}}\right)^{1 / 2}\left(\frac{\mathrm{d} t_{2}}{\mathrm{~d} z_{2}}\right)^{1 / 2}
\end{aligned}
$$




$$
\begin{aligned}
& \propto\left(t_{\infty}-t_{1}\right)^{-1 / 2}\left(t_{\infty}-t_{2}\right)^{1 / 2}\left(t_{\infty}-t_{0}\right)^{-1 / 2}\left(\bar{t}_{\infty}-\bar{t}_{0}\right)^{-1 / 2}\left(t_{1}-t_{2}\right)^{-1}\left(t_{1}-t_{0}\right)^{1 / 2}\left(t_{2}-t_{0}\right)^{-1 / 2} \\
& \quad \times\left(\frac{\mathrm{d} t_{1}}{\mathrm{~d} z_{1}}\right)^{1 / 2}\left(\frac{\mathrm{d} t_{2}}{\mathrm{~d} z_{2}}\right)^{1 / 2} \\
& \rightarrow t_{\infty}^{-1 / 2} \bar{t}_{\infty}^{-1 / 2}\left(t_{1}-t_{2}\right)^{-1} t_{1}^{1 / 2} t_{2}^{-1 / 2}\left(\frac{\mathrm{d} t_{1}}{\mathrm{~d} z_{1}}\right)^{1 / 2}\left(\frac{\mathrm{d} t_{2}}{\mathrm{~d} z_{2}}\right)^{1 / 2}, \quad\left(t_{\infty} \rightarrow \infty, t_{0} \rightarrow 0\right) . \quad \text { (A.36) }
\end{aligned}
$$

$S_{L}$ is the Liouville action as explained at the end of section A.2, which is an irrelevant factor for our purpose and was dropped. Now rewrite $t$ in terms of $z$ using $t \propto z^{1 / n}$. The factor $t_{\infty}^{-1 / 2} \bar{t}_{\infty}^{-1 / 2}$, along with the dropped Liouville factor $\mathrm{e}^{S_{L}}$ and the normalization constants of the twist operators, corresponds in the Lorentzian signature simply to the phase $\mathrm{e}^{-\mathrm{i} E t}$ due to the initial and final states $\sigma_{n}^{--}$. Thus they are irrelevant and we will drop this factor henceforth. The result is

$$
G_{z}=\left\langle\Psi_{A}^{+}\left(z_{1}\right)^{\dagger} \Psi_{B}^{+}\left(z_{2}\right)\right\rangle_{\sigma_{n}^{--}} \propto \frac{1}{\left(z_{1} z_{2}\right)^{1 / 2}\left[1-\left(z_{2} / z_{1}\right)^{1 / n}\right]}
$$

Passing to the cylinder coordinate $w$ by $z=\mathrm{e}^{-\mathrm{i} w}$,

$\left\langle\Psi_{A}^{+}\left(w_{1}\right)^{\dagger} \Psi_{B}^{+}\left(w_{2}\right)\right\rangle_{\sigma_{n}^{--}}=\left\langle\Psi_{A}^{+}\left(z_{1}\right)^{\dagger} \Psi_{B}^{+}\left(z_{2}\right)\right\rangle_{\sigma_{n}^{--}}\left(\frac{\mathrm{d} z_{1}}{\mathrm{~d} w_{1}}\right)^{1 / 2}\left(\frac{\mathrm{d} z_{2}}{\mathrm{~d} w_{2}}\right)^{1 / 2} \propto \frac{1}{1-\mathrm{e}^{\mathrm{i} w / n}}$,

where $w=w_{1}-w_{2}$. As before, the copy labels $A, B$ mean that $w_{1}, w_{2}$ must be understood as $w_{1}+2 \pi(A-1), w_{2}+2 \pi(B-1)$, respectively. Therefore, more precisely,

$\left\langle\Psi_{A}^{+}\left(w_{1}\right)^{\dagger} \Psi_{B}^{+}\left(w_{2}\right)\right\rangle_{\sigma_{n}^{--}}=\frac{\mathrm{i}}{n\left[1-\mathrm{e}^{\mathrm{i}(w+2 \pi(A-B)) / n}\right]}=\frac{\mathrm{e}^{\mathrm{i}(w+2 \pi(A-B)) / 2 n}}{2 n \sin \frac{w+2 \pi(A-B)}{2 n}}$,

where the normalization was fixed by requiring $\Psi_{A}^{+}\left(w_{1}\right)^{\dagger} \Psi_{B}^{+}\left(w_{2}\right) \sim \delta_{A B} / w$.

Similarly, one can compute other correlators of the $S U(2)_{R}$ doublet fields $\Psi_{A}^{s}$ as

$\begin{aligned}\left\langle\Psi_{A}^{s^{\prime}}\left(w_{1}\right)^{\dagger} \Psi_{B}^{s^{\prime}}\left(w_{2}\right)\right\rangle_{\sigma_{n}^{\widetilde{s}}}=\frac{\mathrm{e}^{\mathrm{i} s s^{\prime} w / 2 n}}{2 n \sin \frac{w}{2 n}}, & \left\langle\Psi_{A}^{s^{\prime}}\left(w_{1}\right)^{\dagger} \Psi_{B}^{s^{\prime}}\left(w_{2}\right)\right\rangle_{\sigma_{n}^{\tilde{\alpha} \widetilde{\beta}}}=\frac{\mathrm{e}^{\mathrm{i} \widetilde{\alpha} w / 2 n}}{2 n \sin \frac{w}{2 n}} \\ \left\langle\Psi_{A}^{s^{\prime}}\left(w_{1}\right)^{\dagger} \Psi_{B}^{s^{\prime}}\left(w_{2}\right)\right\rangle_{\tau_{n}^{\widetilde{\alpha}}}=\frac{\mathrm{e}^{\mathrm{i} \widetilde{\alpha} w / 2 n}}{2 n \sin \frac{w}{2 n}}, & \left\langle\Psi_{A}^{s^{\prime}}\left(w_{1}\right)^{\dagger} \Psi_{B}^{s^{\prime}}\left(w_{2}\right)\right\rangle_{\tau_{n}^{s \widetilde{\alpha}}}=\frac{\mathrm{e}^{\mathrm{i} s \widetilde{s} w / 2 n}}{2 n \sin \frac{w}{2 n}},\end{aligned}$

where it is understood that $w$ really means $w+2 \pi(A-B)$; or, in terms of the $\widetilde{S U(2)_{I}}$ doublet fields $\Phi_{A}^{\widetilde{\alpha}}$ defined in (A.9),

$$
\begin{aligned}
\left\langle\Phi_{A}^{\widetilde{\alpha}}\left(w_{1}\right)^{\dagger} \Phi_{B}^{\widetilde{\alpha}}\left(w_{2}\right)\right\rangle_{\sigma_{n}^{\widetilde{s}}}=\frac{\mathrm{e}^{\mathrm{i} s w / 2 n}}{2 n \sin \frac{w}{2 n}}, & \left\langle\Phi_{A}^{\widetilde{\alpha}}\left(w_{1}\right)^{\dagger} \Phi_{B}^{\widetilde{\alpha}}\left(w_{2}\right)\right\rangle_{\sigma_{n}^{\tilde{\beta} \widetilde{\gamma}}}=\frac{\mathrm{e}^{\mathrm{i} \widetilde{\alpha} \widetilde{\beta} w / 2 n}}{2 n \sin \frac{w}{2 n}} \\
\left\langle\Phi_{A}^{\widetilde{\alpha}}\left(w_{1}\right)^{\dagger} \Phi_{B}^{\widetilde{\alpha}}\left(w_{2}\right)\right\rangle_{\tau_{n}^{\tilde{\beta} \widetilde{s}}}=\frac{\mathrm{e}^{\mathrm{i} \widetilde{\alpha} \widetilde{\beta} w / 2 n}}{2 n \sin \frac{w}{2 n}}, & \left\langle\Phi_{A}^{\widetilde{\alpha}}\left(w_{1}\right)^{\dagger} \Phi_{B}^{\widetilde{\alpha}}\left(w_{2}\right)\right\rangle_{\tau_{n}^{s \tilde{\beta}}}=\frac{\mathrm{e}^{\mathrm{i} s w / 2 n}}{2 n \sin \frac{w}{2 n}} .
\end{aligned}
$$

Just as we remarked after the derivation of the bosonic correlator (A.33), these results for fermionic correlators express the fact that the effective length of the CFT cylinder has increased by a factor of $n$ due to the permutation, and the results could have been obtained from this property alone.

\section{Appendix B. Statistical mechanics of the ensemble with $J \neq 0$}

In this appendix, we study the statistical mechanics of the ensemble with $J \neq 0$ studied in section 3.3 . 
For the special case $N_{B}=24, n_{B}=1, N_{F}=n_{F}=0$, i.e., for the D1-D5 system on $\mathrm{K} 3$, state counting was first studied in [50] from the heterotic dual viewpoint. More recently, statistical mechanics of the K3 case was analysed in [21], and the following discussion is a more detailed and generalized version of the one presented in [21].

For the canonical ensemble of bosons and fermions with the spin assignment (3.6), the partition function is

$$
\begin{aligned}
Z(\beta, \mu) & =\operatorname{Tr}\left[\mathrm{e}^{-\beta(N-\mu J)}\right]=\prod_{n=1}^{\infty} \frac{\left[\left(1+z^{1 / 2} q^{n}\right)\left(1+z^{-1 / 2} q^{n}\right)\right]^{n_{F}}\left(1+q^{n}\right)^{N_{F}-2 n_{F}}}{\left[\left(1-z q^{n}\right)\left(1-z^{-1} q^{n}\right)\right]^{n_{B}}\left(1-q^{n}\right)^{N_{B}-2 n_{B}}} \\
& =2^{n_{B}-\frac{N_{F}}{2}} q^{\frac{N_{B}-N_{F}}{24}} \eta(\tau)^{-N_{B}+3 n_{B}-\frac{N_{F}}{2}}\left[\frac{\vartheta_{2}\left(\frac{v}{2} \mid \tau\right)}{\cos \frac{\pi \nu}{2}}\right]^{n_{F}}\left[\frac{\sin \pi \nu}{\vartheta_{1}(\nu \mid \tau)}\right]^{n_{B}} \vartheta_{2}(0 \mid \tau)^{\frac{N_{F}}{2}-n_{F}} .
\end{aligned}
$$

Here $q=\mathrm{e}^{2 \pi \mathrm{i} \tau}=\mathrm{e}^{-\beta}, z=\mathrm{e}^{2 \pi \mathrm{i} \nu}=\mathrm{e}^{\beta \mu}$. After modular transformation, one obtains the expression for $\beta \ll 1$,

$Z(\beta, \mu)=2^{-\frac{N_{F}}{2}} \mathrm{e}^{\frac{\pi^{2} c}{6 \beta}-\frac{\beta}{24}\left(N_{B}-N_{F}\right)-\frac{\mu^{2} \beta}{2}\left(n_{B}-\frac{n_{F}}{4}\right)}\left(\frac{\beta}{2 \pi}\right)^{\frac{N_{B}}{2}-n_{B}}\left[\frac{\sinh \frac{\beta \mu}{2}}{\sin \pi \mu}\right]^{n_{B}} \frac{1}{\left[\cosh \frac{\beta \mu}{4}\right]^{n_{F}}}$

up to exponentially suppressed terms by $\mathrm{e}^{-\frac{2 \pi^{2}}{\beta}}$. Here, $c \equiv N_{B}+N_{F} / 2$. For the 'Hamiltonian' $N-\mu J$ to be positive definite, we must restrict the range of the chemical potential to $|\mu|<1$. Therefore, for $\beta \ll 1$, one can further simplify (B.2) as

$$
Z(\beta, \mu) \sim \beta^{\frac{N_{B}}{2}}\left(\frac{\mu}{\sin \pi \mu}\right)^{n_{B}} \mathrm{e}^{\pi^{2} c / 6 \beta},
$$

up to a numerical factor. Note that this result does not depend on $n_{F}$; all spins are carried by bosons. This is because the Pauli exclusion principle exacts a high price in $N$ when the fermions carry a macroscopic amount of angular momentum.

Let us compute the entropy of this system by thermodynamic approximation, i.e., by saddle point approximation. By the standard formula of thermodynamics,

$N=-\left(\frac{\partial \log Z}{\partial \beta}\right)_{\beta \mu}=\frac{c \pi^{2}}{6 \beta^{2}}+\frac{n_{B} \mu}{\beta} g(\mu), \quad J=\left(\frac{\partial \log Z}{\partial(\beta \mu)}\right)_{\beta}=\frac{n_{B}}{\beta} g(\mu)$,

where

$$
g(\mu) \equiv \frac{1}{\mu}-\frac{\pi}{\tan \pi \mu}=\frac{\pi^{2} \mu}{3}+\frac{\pi^{4} \mu^{3}}{45}+\cdots .
$$

In deriving (B.4), we ignored $\beta^{N_{B} / 2}$ in (B.3) in the thermodynamic approximation. The entropy is

$S=\beta(N-\mu J-F)=\beta(N-\mu J)+\log Z=\frac{c \pi^{2}}{3 \beta}+n_{B} \log \left(\frac{\mu}{\sin \pi \mu}\right)$.

From (B.4), (B.6), we obtain

$$
\begin{aligned}
& S=2 \pi \sqrt{\frac{c}{6}(N-\mu J)}+n_{B} \log \left(\frac{\mu}{\sin \pi \mu}\right), \\
& J=\frac{3 n_{B}^{2} \mu g(\mu)^{2}}{c \pi^{2}}\left[\sqrt{1+\frac{2 c N \pi^{2}}{3 \mu^{2} n_{B}^{2} g(\mu)^{2}}}-1\right] .
\end{aligned}
$$


From relation (B.8) and the form of the function $g(\mu)(\mathrm{B} .5)$, it is easy to see that $\mu \rightarrow \operatorname{sign}(J)$ is needed in order that $|J|=\mathcal{O}(N)$. More precisely, we need $|\mu-\operatorname{sign}(J)| \sim N^{-1 / 2}$. Therefore,

$$
S=\log d_{N, J} \approx 2 \pi \sqrt{\frac{c}{6}(N-|J|)},
$$

where we dropped the subleading log term coming from the second term in (B.7). Note that $\mu \rightarrow \pm 1$ implies that the energy of bosons with $J= \pm 1$ vanishes and Bose-Einstein condensation of those bosons occurs.

\section{References}

[1] Maldacena J M 1998 The large $N$ limit of superconformal field theories and supergravity Adv. Theor. Math. Phys. 2231

Maldacena J M 1999 Int. J. Theor. Phys. 381113 (Preprint hep-th/9711200)

[2] Witten E 1998 Anti-de Sitter space and holography Adv. Theor. Math. Phys. 2253 (Preprint hep-th/9802150)

[3] Gubser S S, Klebanov I R and Polyakov A M 1998 Gauge theory correlators from non-critical string theory Phys. Lett. B 428105 (Preprint hep-th/9802109)

[4] Maldacena J M 2003 Eternal black holes in anti-de-Sitter J. High Energy Phys. JHEP04(2003)021 (Preprint hep-th/0106112)

[5] Hemming S, Keski-Vakkuri E and Kraus P 2002 Strings in the extended BTZ spacetime J. High Energy Phys. JHEP10(2002)006 (Preprint hep-th/0208003)

[6] Kraus P, Ooguri H and Shenker S 2003 Inside the horizon with AdS/CFT Phys. Rev. D 67124022 (Preprint hep-th/0212277)

[7] Levi T S and Ross S F 2003 Holography beyond the horizon and cosmic censorship Phys. Rev. D 68044005 (Preprint hep-th/0304150)

[8] Fidkowski L, Hubeny V, Kleban M and Shenker S 2004 The black hole singularity in AdS/CFT J. High Energy Phys. JHEP02(2004)014 (Preprint hep-th/0306170)

[9] Barbon J L F and Rabinovici E 2003 Very long time scales and black hole thermal equilibrium J. High Energy Phys. JHEP11(2003)047 (Preprint hep-th/0308063)

[10] Balasubramanian V and Levi T S 2004 Beyond the veil: inner horizon instability and holography Phys. Rev. D 70106005 (Preprint hep-th/0405048)

[11] Kleban M, Porrati M and Rabadan R 2004 Poincare recurrences and topological diversity J. High Energy Phys. JHEP10(2004)030 (Preprint hep-th/0407192)

[12] Festuccia G and Liu H 2005 Excursions beyond the horizon: black hole singularities in Yang-Mills Preprint hep-th/0506202

[13] Behrndt K, Lopes Cardoso G, de Wit B, Lust D, Mohaupt T and Sabra W A 1998 Higher-order black-hole solutions in $N=2$ supergravity and Calabi-Yau string backgrounds Phys. Lett. B 429289 (Preprint hepth/9801081)

Lopes Cardoso G, de Wit B and Mohaupt T 1999 Corrections to macroscopic supersymmetric black-hole entropy Phys. Lett. B 451309 (Preprint hep-th/9812082)

Lopes Cardoso G, de Wit B and Mohaupt T 2000 Macroscopic entropy formulae and non-holomorphic corrections for supersymmetric black holes Nucl. Phys. B 56787 (Preprint hep-th/9906094)

Cardoso G L, de Wit B, Kappeli J and Mohaupt T 2004 Asymptotic degeneracy of dyonic $N=4$ string states and black hole entropy J. High Energy Phys. JHEP12(2004)075 (Preprint hep-th/0412287)

[14] Ooguri H, Strominger A and Vafa C 2004 Black hole attractors and the topological string Phys. Rev. D 70 106007 (Preprint hep-th/0405146)

[15] Dabholkar A 2004 Exact counting of black hole microstates Preprint hep-th/0409148

[16] Dabholkar A, Kallosh R and Maloney A 2004 A stringy cloak for a classical singularity J. High Energy Phys. JHEP12(2004)059 (Preprint hep-th/0410076)

[17] Dabholkar A, Denef F, Moore G W and Pioline B 2005 Exact and asymptotic degeneracies of small black holes Preprint hep-th/0502157

Dabholkar A, Denef F, Moore G W and Pioline B 2005 Precision counting of small black holes Preprint hep-th/0507014

[18] Hubeny V, Maloney A and Rangamani M 2005 String-corrected black holes J. High Energy Phys. JHEP05(2005)035 (Preprint hep-th/0411272)

[19] Sen A 2005 Black holes, elementary strings and holomorphic anomaly Preprint hep-th/0502126 
Sen A 2005 Black holes and the spectrum of half-BPS states in $N=4$ supersymmetric string theory Preprint hep-th/0504005

Sen A 2005 Stretching the horizon of a higher dimensional small black hole Preprint hep-th/0505122

Sen A 2005 Black hole entropy function and the attractor mechanism in higher derivative gravity Preprint hep-th/0506177

[20] Kraus P and Larsen F 2005 Microscopic black hole entropy in theories with higher derivatives Preprint hepth/0506176

[21] Iizuka N and Shigemori M 2005 A note on D1-D5-J system and 5D small black ring Preprint hep-th/0506215

[22] Lunin O and Mathur S D 2002 AdS/CFT duality and the black hole information paradox Nucl. Phys. B 623 342 (Preprint hep-th/0109154)

Lunin O and Mathur S D 2002 Statistical interpretation of Bekenstein entropy for systems with a stretched horizon Phys. Rev. Lett. 88211303 (Preprint hep-th/0202072)

Mathur S D 2005 The fuzzball proposal for black holes: An elementary review Preprint hep-th/0502050

[23] Mathur S D, Saxena A and Srivastava Y K 2004 Constructing 'hair' for the three charge hole Nucl. Phys. B 680 415 (Preprint hep-th/0311092)

Lunin O 2004 Adding momentum to D1-D5 system J. High Energy Phys. JHEP04(2004)054 (Preprint hepth/0404006)

Giusto S, Mathur S D and Saxena A 2004 Dual geometries for a set of 3-charge microstates Nucl. Phys. B 701 357 (Preprint hep-th/0405017)

Giusto S, Mathur S D and Saxena A 2005 3-charge geometries and their CFT duals Nucl. Phys. B 710425 (Preprint hep-th/0406103)

Giusto S and Mathur S D 2004 Geometry of D1-D5-P bound states Preprint hep-th/0409067

[24] Bena I and Kraus P 2004 Three charge supertubes and black hole hair Phys. Rev. D 70046003 (Preprint hep-th/0402144)

[25] Bena I and Kraus P 2005 Microstates of the D1-D5-KK system Phys. Rev. D 72025007 (Preprint hepth/0503053)

[26] Jejjala V, Madden O, Ross S F and Titchener G 2005 Non-supersymmetric smooth geometries and D1-D5-P bound states Phys. Rev. D 71124030 (Preprint hep-th/0504181)

[27] Bena I and Warner N P 2005 Bubbling supertubes and foaming black holes Preprint hep-th/0505166

[28] Berglund P, Gimon E G and Levi T S 2005 Supergravity microstates for BPS black holes and black rings Preprint hep-th/0505167

[29] Taylor M 2005 General 2 charge geometries Preprint hep-th/0507223

[30] Banados M, Teitelboim C and Zanelli J 1992 The black hole in three-dimensional space-time Phys. Rev. Lett. 691849 (Preprint hep-th/9204099)

Banados M, Henneaux M, Teitelboim C and Zanelli J 1993 Geometry of the (2+1) black hole Phys. Rev. D 48 1506 (Preprint gr-qc/9302012)

[31] Corley S, Jevicki A and Ramgoolam S 2002 Exact correlators of giant gravitons from dual $N=4$ SYM theory Adv. Theor. Math. Phys. 5809 (Preprint hep-th/0111222)

[32] Berenstein D 2004 A toy model for the AdS/CFT correspondence J. High Energy Phys. JHEP07(2004)018 (Preprint hep-th/0403110)

[33] Lin H, Lunin O and Maldacena J 2004 Bubbling AdS space and 1/2 BPS geometries J. High Energy Phys. JHEP10(2004)025 (Preprint hep-th/0409174)

[34] Balasubramanian V, Jejjala V and Simon J 2005 The library of Babel Preprint hep-th/0505123

Balasubramanian V, de Boer J, Jejjala V and Simon J 2005 The library of Babel: on the origin of gravitational thermodynamics Preprint hep-th/0508023

[35] Rey S J and Hikida Y 2005 5d black hole as emergent geometry of weakly interacting 4d hot Yang-Mills gas Preprint hep-th/0507082

Berenstein D 2005 Large $N$ BPS states and emergent quantum gravity Preprint hep-th/0507203

Shepard P G 2005 Black hole statistics from holography Preprint hep-th/0507260

Silva P J 2005 Rational foundation of GR in terms of statistical mechanic in the AdS/CFT framework Preprint hep-th/0508081

[36] Lunin O, Maldacena J and Maoz L 2002 Gravity solutions for the D1-D5 system with angular momentum Preprint hep-th/0212210

[37] Balasubramanian V, de Boer J, Keski-Vakkuri E and Ross S F 2001 Supersymmetric conical defects: towards a string theoretic description of black hole formation Phys. Rev. D 64064011 (Preprint hep-th/0011217)

[38] Maldacena J M and Maoz L 2002 De-singularization by rotation J. High Energy Phys. JHEP12(2002)055 (Preprint hep-th/0012025)

[39] Strominger A and Vafa C 1996 Microscopic origin of the Bekenstein-Hawking entropy Phys. Lett. B 37999 (Preprint hep-th/9601029) 
[40] de Boer J 1999 Six-dimensional supergravity on S**3 $\times \operatorname{AdS}(3)$ and 2d conformal field theory Nucl. Phys. B 548139 (Preprint hep-th/9806104)

[41] Seiberg N and Witten E 1999 The D1/D5 system and singular CFT J. High Energy Phys. JHEP04(1999)017 (Preprint hep-th/9903224)

[42] Larsen F and Martinec E J 1999 U(1) charges and moduli in the D1-D5 system J. High Energy Phys. JHEP06(1999)019 (Preprint hep-th/9905064)

[43] Strominger A 1998 Black hole entropy from near-horizon microstates J. High Energy Phys. JHEP02(1998)009 (Preprint hep-th/9712251)

[44] Lunin O and Mathur S D 2001 Metric of the multiply wound rotating string Nucl. Phys. B 61049 (Preprint hep-th/0105136)

[45] Callan C G, Maldacena J M and Peet A W 1996 Extremal black holes as fundamental strings Nucl. Phys. B 475 645 (Preprint hep-th/9510134)

[46] Dabholkar A, Gauntlett J P, Harvey J A and Waldram D 1996 Strings as solitons and black holes as strings Nucl. Phys. B 47485 (Preprint hep-th/9511053)

[47] Horowitz G T and Tseytlin A A 1995 A new class of exact solutions in string theory Phys. Rev. D 512896 (Preprint hep-th/9409021)

[48] Tseytlin A A 1996 Generalised chiral null models and rotating string backgrounds Phys. Lett. B 38173 (Preprint hep-th/9603099)

[49] Keski-Vakkuri E 1999 Bulk and boundary dynamics in BTZ black holes Phys. Rev. D 59104001 (Preprint hep-th/9808037)

[50] Russo J G and Susskind L 1995 Asymptotic level density in heterotic string theory and rotating black holes Nucl. Phys. B 437611 (Preprint hep-th/9405117)

[51] Elvang H, Emparan R, Mateos D and Reall H S 2004 A supersymmetric black ring Phys. Rev. Lett. 93211302 (Preprint hep-th/0407065)

Elvang H, Emparan R, Mateos D and Reall H S 2005 Supersymmetric black rings and three-charge supertubes Phys. Rev. D 71024033 (Preprint hep-th/0408120)

[52] Lunin O, Mathur S D and Saxena A 2003 What is the gravity dual of a chiral primary? Nucl. Phys. B 655185 (Preprint hep-th/0211292)

[53] Boni M and Silva P J 2005 Revisiting the D1/D5 system or bubbling in AdS(3) Preprint hep-th/0506085

[54] Bena I and Warner N P 2004 One ring to rule them all ... and in the darkness bind them? Preprint hep-th/0408106

[55] Bena I and Kraus P 2004 Microscopic description of black rings in AdS/CFT J. High Energy Phys. JHEP12(2004)070 (Preprint hep-th/0408186)

[56] Giusto S and Mathur S D 2004 Fuzzball geometries and higher derivative corrections for extremal holes Preprint hep-th/0412133

[57] Hamidi S and Vafa C 1987 Interactions on orbifolds Nucl. Phys. B 279465

[58] Dixon L J, Friedan D, Martinec E J and Shenker S H 1987 The conformal field theory of orbifolds Nucl. Phys. B 28213

[59] Bantay P 1998 Characters and modular properties of permutation orbifolds Phys. Lett. B 419175 (Preprint hep-th/9708120)

[60] Arutyunov G E and Frolov S A 1998 Virasoro amplitude from the S(N) R**24 orbifold sigma model Theor. Math. Phys. 11443 (Preprint hep-th/9708129)

Arutyunov G E and Frolov S A 1998 Four graviton scattering amplitude from S(N) R**8 supersymmetric orbifold sigma model Nucl. Phys. B 524159 (Preprint hep-th/9712061)

Arutyunov G, Frolov S and Polishchuk A 1999 On Lorentz invariance and supersymmetry of four particle scattering amplitudes in S(N) R**8 orbifold sigma model Phys. Rev. D 60066003 (Preprint hep-th/9812119)

[61] Evslin J, Halpern M B and Wang J E 1999 General Virasoro construction on orbifold affine algebra Int. J. Mod. Phys. A 144985 (Preprint hep-th/9904105)

de Boer J, Evslin J, Halpern M B and Wang J E 2000 New duality transformations in orbifold theory Int. J. Mod. Phys. A 151297 (Preprint hep-th/9908187)

Halpern M B and Wang J E 2001 More about all current-algebraic orbifolds Int. J. Mod. Phys. A 1697 (Preprint hep-th/0005187)

[62] Jevicki A, Mihailescu M and Ramgoolam S 2000 Gravity from CFT on S**N(X): symmetries and interactions Nucl. Phys. B 57747 (Preprint hep-th/9907144)

[63] Mihailescu M 2000 Correlation functions for chiral primaries in $D=6$ supergravity on $\operatorname{AdS}(3) \times \mathrm{S}(3) \mathrm{J}$. High Energy Phys. JHEP02(2000)007 (Preprint hep-th/9910111)

[64] Lunin O and Mathur S D 2001 Correlation functions for M(N)/S(N) orbifolds Commun. Math. Phys. 219399 (Preprint hep-th/0006196)

Lunin O and Mathur S D 2001 Correlation functions for orbifolds of the type M(N)/S(N) Nucl. Phys. Proc. Suppl. 101296 
[65] Argurio R, Giveon A and Shomer A 2000 Superstrings on AdS(3) and symmetric products J. High Energy Phys. JHEP12(2000)003 (Preprint hep-th/0009242)

[66] Lunin O and Mathur S D 2002 Three-point functions for $\mathrm{M}(\mathrm{N}) / \mathrm{S}(\mathrm{N})$ orbifolds with $N=4$ supersymmetry Commun. Math. Phys. 227385 (Preprint hep-th/0103169)

[67] de Boer J, Halpern M B and Obers N A 2001 The operator algebra and twisted KZ equations of WZW orbifolds J. High Energy Phys. JHEP10(2001)011 (Preprint hep-th/0105305)

[68] David J R, Mandal G and Wadia S R 2002 Microscopic formulation of black holes in string theory Phys. Rep. 369549 (Preprint hep-th/0203048)

[69] Wadia S R 2000 Lectures on the microscopic modeling of the 5-dim black hole of IIB string theory and the D1/D5 system Preprint hep-th/0006190

[70] Maldacena J M, Moore G W and Strominger A 1999 Counting BPS black holes in toroidal type II string theory Preprint hep-th/9903163

[71] Dijkgraaf R, Vafa C, Verlinde E and Verlinde H 1989 The operator algebra of orbifold models Commun. Math. Phys. 123485

[72] Witten E 1982 Constraints on supersymmetry breaking Nucl. Phys. B 202253

[73] Vafa C and Witten E 1994 A strong coupling test of S duality Nucl. Phys. B 4313 (Preprint hep-th/9408074) 